\title{
Sliding Mode Control Applied to Reconfigurable Flight Control Design
}

\author{
R.A. Hess' ${ }^{\prime}$ and S.R. Wells ${ }^{2}$ \\ Dept. of Mechanical and Aeronautical Engineering \\ One Shields Ave. \\ University of California \\ Davis, CA 95616-5294
}

\begin{abstract}
Sliding mode control is applied to the design of a flight control system capable of operating with limited bandwidth actuators and in the presence of significant damage to the airframe and/or control effector actuators. Although inherently robust, sliding mode control algorithms have been hampered by their sensitivity to the effects of parasitic unmodeled dynamics, such as those associated with actuators and structural modes. It is known that asymptotic observers can alleviate this sensitivity while still allowing the system to exhibit significant robustness. This approach is demonstrated. The selection of the sliding manifold as well as the interpretation of the linear design that results after introduction of a boundary layer is accomplished in the frequency domain. The design technique is exercised on a pitch-axis controller for a simple short-period model of the High Angle of Attack F-18 vehicle via computer simulation. Stability and performance is compared to that of a system incorporating a controller designed by classical loop-shaping techniques.
\end{abstract}

\footnotetext{
1 Professor and Vice Chairman, Associate Fellow AIAA, rahess@ucdavis.edu

${ }^{2}$ Graduate Student, Major, United States Air Force, Student Member AIAA
} 


\section{Introduction}

\section{Sliding Mode Control}

Sliding Mode Control (SMC) is an approach to the design of control systems that exhibit robust performance in the presence of large uncertainties in plant dynamics. The basic concepts of SMC first appeared in the Russian literature in the early 1930's. In the mid 1970's sliding mode control concepts began to appear in the western literature when a text by Itkis $^{1}$ and a paper by Utkin ${ }^{2}$ were published in English. A number of textbooks ${ }^{3,4}$ devoted solely to SMC have since appeared as well as a vast array of papers too numerous to list. By 1993, general application areas included: robotic control, motor control, aircraft and spacecraft control, flexible structure control, and power systems. ${ }^{5}$ Current applications also include: controlling the convergence rates for neural net learning algorithms; ${ }^{6}$ direct robust exact differentiation; ${ }^{7}$ missile autopilot; ${ }^{8}$ control of multiple unmanned air vehicles in close-formation flight; ${ }^{9}$ and reconfigurable flight control. ${ }^{10}$

A reconfigurable flight control system is one that is able to compensate for sudden, potentially large, unknown failure events in real-time using on-line adaptive control laws and/or adaptive redistribution of control. Of all the robust control schemes in the current literature, SMC appears to be among the most promising for handling sudden, large changes in vehicle dynamics due to failures. This is attributable to the fact that SMC can demonstrate invariance to so-called matched uncertainty. Plant uncertainties are defined as matched when they lie in the image of the plant input matrix, i.e. the uncertainties affect the plant dynamics only through the plant input channels. Thus, if the system is invariant in the presence of uncertainties such as those arising from airframe or actuator damage, there exists no need to perform failure detection, system identification, and on-line control algorithm redesign in applying SMC to reconfigurable flight control. Indeed, it was this potential simplicity that motivated the research to be described. 
Strictly speaking, SMC designs are not reconfigurable, i.e., the control system architecture is not changed when failure or damage occurs. Nonetheless, the terminology will be retained herein, as the design approach is intended to serve as an alternative to truly reconfigurable systems.

Excellent review articles and surveys regarding SMC theory and applications are available, e.g. Refs. 2,5,11-15. Hence only a brief, simplified tutorial overview is given here, with emphasis on implementation and design issues.

\section{Overview of SMC}

There are several key properties of a sliding mode controller that make it very attractive for controlling an uncertain system. These properties are well known and are reviewed here without proof. 5

While on the sliding mode, the system dynamics are invariant to matched uncertainty.

The hypersurface that describes $\sigma=0$ defines the transient response of the system during the sliding mode.

While on the sliding mode, the trajectory dynamics are of a lower order than the original model. Consider the uncertain system with $m$ inputs and $n$ states given by:

$$
\dot{\mathbf{x}}(\mathrm{t})=\mathbf{A}(\mathbf{x}, \mathrm{t})+\mathbf{B}(\mathbf{x}, \mathrm{t}) \mathbf{u}(\mathrm{t})+f(\mathrm{t}, \mathbf{x}, \mathbf{u})
$$

where $f$ represents the parameter uncertainties present in the system. The vector $f$ is assumed to be unknown but bounded by some known function of time, system state and control vectors. In simple terms, the objective of SMC is to define

a.) $m$ sliding surfaces or manifolds, represented in vector form as $\sigma(\mathbf{x})=\mathbf{0}$, and

b.) a variable structure control given by

$$
\mathbf{u}(\mathbf{x}, t)=\rho \operatorname{sgn}(\sigma)
$$


such that the system is driven to the sliding surface $\sigma=0$ in finite time and remains upon this surface for all subsequent time. When $\sigma=\mathbf{0}$, a sliding mode is said to have been obtained. Equation ( 2 ) is said to describe variable structure control because the control structure is dependent upon the sign of the function $\sigma$.

This problem statement implies a two-step design process. First, the sliding manifold(s) must be designed. This can be accomplished by a wide variety of approaches ranging from arbitrarily selecting desired error dynamics to an LQR-like design utilizing the state equations in the so-called regular form ${ }^{4}$. A different approach utilizing frequency domain methods is offered in this work. The second step is the selection of $\rho$ such that the sliding manifold is "attractive"--this is known as the reaching condition. One of the most common methods to prove the reaching condition is the use of Lyapunov stability criteria with $\mathrm{V}=\boldsymbol{\sigma}^{\mathrm{T}} \boldsymbol{\sigma}$ as the Lyapunov function. These proofs can become very involved, especially when parameter variations are included. Even if global analytic stability bounds can be obtained, choosing $\rho$ analytically can be problematic. This is due to the fact that not only must bounds on $f$ be known, but also bounds on desired values of the state vector, if model reference control is desired. Estimating the latter bound is especially difficult if there is an outer loop being closed by another agent, such as a human pilot in a flight control system.

\section{Implementation Issues}

When the system is on the sliding surface $(\sigma=0)$, the control defined by Eqn. ( 2 ) is not defined and the elements of the control vector $\mathbf{u}(\mathbf{x}, \mathbf{t})$ will oscillate at infinite frequency. This is sometimes referred to in the literature as "chattering." Others, however, would argue that chattering occurs when noise or delays appear in the switching logic resulting in high frequency oscillations in the state variables as well as the elements of $u(x, t)$. The infinite frequency switching of a pure SMC is expected and results in smooth state trajectories. Nonetheless, this 
infinite frequency switching in $\mathbf{u}(\mathbf{x}, \mathbf{t})$ can not be obtained in practice, except in limited applications. One of the simplest and most common methods for eliminating the infinite frequency switching in the control signal is the use of a so-called boundary layer near the sliding manifolds. This is typically accomplished by replacing the signum function of the switching control law of Eqn. (2) with saturation elements. The slope of the linear portion of the saturation element is denoted as $(1 / \varepsilon$,$) with \varepsilon$ being the thickness of the boundary layer. The saturation elements result in a continuous control signal, however the state trajectories are no longer constrained to the sliding manifold - only to the boundary layer near it. The price of achieving this continuous control is the loss of the invariance property. Nonetheless, considerable robustness can remain with the SMC structure when boundary layers are utilized. Strictly speaking, the resulting system should be referred to as a pseudo-sliding mode controller, since true sliding mode behavior will not be in evidence. However, for the sake of simplicity, the SMC system with boundary layer is referred to here as an SMC system.

Even when a boundary layer is used, SMC systems are vulnerable to the effects of so-called parasitic dynamics, i.e., high-frequency dynamics often neglected in control system design. In flight control applications, such neglected elements may include those associated with actuation systems and structural modes. These neglected elements can also be considered equivalent to unstructured uncertainty in the plant model. Although it has been suggested that boundary layers alone can accommodate the effects of these neglected dynamics, ${ }^{16}$ it has been demonstrated that finite-bandwidth actuators/sensors can cause instability within the boundary layer. $^{15}$ One of the most straightforward approaches to address this problem is the use of asymptotic observers. ${ }^{15}$ Figure 1, adopted from Ref. 15 illustrates the rationale behind the use of observers in SMC systems. As the authors of Ref. 15 indicate, the observer essentially serves as a high-frequency bypass loop in the SMC system, effectively shielding the parasitic dynamics 
from the high frequency activity of the controller. The observer also helps mitigate the nonlinear effects of rate and position limiting of actuators--problems which are typically catastrophic to SMC systems.

\section{Interpretation of Linear SMC Design in the Frequency Domain}

Considering a linear plant or vehicle model, the SMC system including boundary layers can be interpreted as a linear system and analyzed in the frequency domain. That is, classical loop-shaping principles can be applied to the design, both in terms of choosing appropriate sliding manifolds and in evaluating the characteristics of the final SMC configuration. Note that, if a boundary layer is employed, and the boundary layer thickness is selected such that the limits of the saturation element are never reached, the control law is essentially a high gain linear controller. For example, consider a sliding function of the form

$$
\sigma=c_{1} \dot{e}+c_{0} e+c_{-1} \int e d t
$$

and an SMC control law (with a boundary layer)

$$
\mathrm{u}=\rho \operatorname{sat}\left(\frac{\sigma}{\varepsilon}\right)
$$

If the saturation element always operates in the linear region, the feedback control law in the Laplace domain looks like a PID controller:

$$
u(s)=\frac{\rho}{\varepsilon} \sigma=\frac{\rho}{\varepsilon}\left(c_{1} s+c_{0}+\frac{c_{-1}}{s}\right) e(s)
$$

This fact can be used to help choose both the sliding function constants $\left(c_{-1}, c_{0}\right.$, and $\left.c_{1}\right)$ and the control law gain $(\rho / \varepsilon)$. This interpretation of the sliding function allows the use of familiar loop shaping techniques and provides insight into the behavior of the controller. This is demonstrated in the example to follow. 


\section{SMC Design Methodology}

A proposed SMC design methodology can now be offered. It is couched in terms of a SISO application, with a brief discussion of extension to MIMO applications to follow. Recalling that the objectives of SMC design are finding appropriate sliding surfaces and switching logic, the procedure to be described approaches this problem from a frequency-domain based, model-reference approach.

(1) The vehicle model is obtained, along with an estimate of the frequency beyond which parasitic dynamics (or unstructured uncertainties) are likely to come into play. This frequency is referred to as the limit frequency in this discussion.

(2) A reference model is chosen. Since piloted flight control is of interest in the present application, this reference model should be selected with an eye towards Level 1 handling qualities with no pilot-induced oscillation (PIO) tendencies. An example of accomplishing this through reference model selection is given in Ref. 17 based upon a pilot model-based handling qualities and PIO prediction technique introduced in Ref.18.

(3) The desired feedback structure of the control system is determined with a square system architecture. For example, if a pitch-rate command flight control system is desired, then pitch rate $\left(q_{c}\right)$ becomes the output of the reference model, and estimated pitch rate $(\hat{q})$ is fed back to the SMC system from the observer. System error is then defined as $e(t)=q_{c}(t)-\hat{q}(t)$. In the SISO format a control distribution matrix is defined if more than one control effector is available. The sliding manifold, $\sigma$ is chosen based upon the following principles:

a) $\sigma$ is derived from a tracking error expression as

$$
\sigma=e(t)^{p-1}+K_{p-2} e(t)^{p-2}+\cdots+K_{0} e(t)+K_{-1} \int e(t) d t
$$


where $\mathrm{p}$ is the relative order of the system, i.e., the number of times the vehicle output must be differentiated for the input to appear. Note that the $(\mathrm{p}-1)^{\mathrm{st}}$ derivative of the error signal is included in the definition of $\sigma$. An integral term also appears in Eqn. (6) to counter the steadystate bias often created with the use of a boundary layer. Excluding the integral term, Eqn. ( 6 ) can be written in a more concise notation ${ }^{16}$ as $\sigma=\left(\frac{d}{d t}+\lambda\right)^{p-1}$ e. This approach allows an efficient and concise description of the error dynamics while in sliding mode. However, the following approach is pursued here:

b) Recognizing that a boundary layer is to be implemented, the control law is expressed as a linear transfer function as discussed earlier.

$$
\mathrm{u}(\mathrm{s})=\frac{\rho}{\varepsilon} \sigma=\mathrm{K}_{\rho}\left(\mathrm{s}^{\mathrm{p}-1}+\mathrm{K}_{\mathrm{p}-2} \mathrm{~s}^{\mathrm{p}-2}+\cdots+\mathrm{K}_{0}+\frac{\mathrm{K}_{-1}}{\mathrm{~s}}\right) \mathrm{e}(\mathrm{s})
$$

The parameters $\mathrm{K}_{\mathrm{i}}$ are chosen to provide desirable properties in the frequency domain. This means creating a loop transmission with broad K/s-like characteristics around crossover. ${ }^{19}$ This will always be possible since enough derivatives are included in Eqn. ( 6 ) to create exact $\mathrm{K} / \mathrm{s}$ characteristics beyond a certain frequency (at least as high as the limit frequency). Parasitic dynamics are deliberately excluded in this formulation. This step will involve obtaining an estimate of $\mathrm{K}_{\rho}$, as this value will determine the crossover frequency of the loop transmission. This crossover frequency is selected to provide acceptable stability margins as obtained from a Bode plot of the loop transmission but using a value of $K_{\rho}$ at least as large as the largest amplitude limit of any of the control effectors. The latter criterion is included to accommodate maximum trim positions of the control effectors. As opposed to typical designs involving loop shaping, very high crossover frequencies may result from this step. Indeed these frequencies may be well beyond the limit frequency. This result is of no immediate concern. 
(4) Using the $\mathrm{K}_{i}$ 's just determined in the definition of the sliding function, the existence of a sliding mode is verified in the inner loop using a true SMC. This step is completed without the observer, actuators, reference model or pilot model, i.e., assuming that no outer-loop is being utilized. If necessary, $\rho$ is increased until sliding behavior is created. The initial value of $\rho=K_{\rho}$ obtained in step (3b) should be considered a lower limit in this process. While an analytical approach to determine $\rho$ is certainly possible here, a more expedient route of establishing the sliding mode using a computer simulation of the system is also possible.

(5) A boundary layer is included in the controller by replacing the control law of Eqn. (2) with the control law of Eqn. ( 4 ). A typical procedure involves beginning with $\varepsilon=1$ and decreasing $\varepsilon$ until high-frequency switching is just avoided. If $\varepsilon>1$ is necessary $\rho$ should be increased in proportion to $\varepsilon$. Again, a simulation of the SMC system is a convenient way of finding this $\varepsilon$. Near-perfect tracking (with a continuous control signal) in the face of large parameter variations should be observed.

(6) Parasitic dynamics are included in the model. The SMC controller will very likely be unstable at this juncture.

(7) An asymptotic observer or state estimator is created via pole-placement. The form of the estimator is given by

$$
\dot{\hat{\mathbf{x}}}=\mathbf{A} \hat{\mathbf{x}}+\mathbf{B u}+\mathbf{G}(\mathbf{y}-\mathbf{C} \hat{\mathbf{x}})
$$

where $^{\wedge}$ denotes "estimate of", $\mathbf{y}$ is the measurement vector, and $\mathbf{G}$ is the matrix of observer gains. The poles or eigenvalues should be chosen to lie within the frequency range bracketed by the limit frequency of step (1) and the bandwidth of the reference model of step (2). The choice of observer eigenvalues is of considerable importance in this formulation. Eigenvalues that are too large will defeat the purpose of the observer and amplify the effects of sensor noise, while eigenvalues that are too small will reduce the robustness of the SMC design. 
(8) The frequency domain characteristics of the open and closed-loop SMC system with observer, boundary layer and reference model are examined to ensure that stability of the linear system is in evidence.

\section{The Design Methodology Applied to Reconfigurable Control}

\section{Vehicle, Sensor Noise and Actuator Models}

The proposed design methodology is exercised next by means of a simple flight control example involving maintenance of system stability and performance robustness in the presence of airframe/actuator. The vehicle model is taken from Ref. 20 and represents the simplified longitudinal dynamics of the NASA High Angle of Attack Research Vehicle (HARV). Longitudinal control is provided by a horizontal stabilator and pitch thrust vectoring. The vehicle, sensor noise and actuator models are given in the appendix. Note that amplitude and rate limits are included in the actuator models. The appendix also includes a model of the vehicle with assumed damage of a $50 \%$ reduction in stabilator area. An approximation for a fuselage bending mode is also included as is the pilot model used in the computer simulation. The selection of a simple vehicle aerodynamic model was deliberate. By doing so, the complete vehicle, actuator, control system and pilot models could be described with a detail that would allow the reader to repeat the computer simulation if so desired.

\section{SMC Design}

Design Steps The SMC design will follow the procedure outline in the preceding discussion.

Figure 2 is a diagrammatic representation of the pilot/vehicle/controller system.

(1) The nominal aircraft model is given in the appendix. A pitch-rate flight control system is desired. Two control effectors are available. A simple control distribution matrix is employed between the single pseudo-control $u(t)$ and the control effector variables $\delta_{s}(t)$ and $\delta_{t}(t)$. Thus, 


$$
\left\{\begin{array}{l}
\delta_{s} \\
\delta_{1}
\end{array}\right\}=\left[\begin{array}{l}
1 \\
1
\end{array}\right] \mathrm{u}(\mathrm{t})
$$

To demonstrate the ability of the SMC system to handle parasitic dynamics, the actuator dynamics and the approximated structural model are ignored in the control design. The limit frequency is selected as $20 \mathrm{rad} / \mathrm{s}$.

(2) The reference model is chosen as

$$
\frac{q_{d}}{q_{c}}(s)=\frac{100}{(s+5)(s+20)}
$$

where $\mathrm{q}_{\mathrm{c}}$ is the pilot input from the cockpit control inceptor (a pitch-rate command) and $\mathrm{q}_{d}$ is the output of the reference model. This model yields acceptable task-independent handling qualities predictions using the pilot modeling technique of Ref. 18 .

a.) The relative order of the vehicle dynamics (sans actuators) is 1 , therefore the sliding manifold is given simply by

$$
\sigma=e(t)+K_{-1} \int e(t) d t
$$

b.) Using loop shaping, the parameter $\mathrm{K}_{-1}$ is selected as: $\mathrm{K}_{-1}=20.0$. A preliminary value of $\rho=-30$, a value equal to the maximum amplitudes of the control effectors, yields excellent gain and phase margins, and a crossover frequency of $250 \mathrm{rad} / \mathrm{s}$ ! The previous caveat regarding high crossover frequencies should be borne in mind at this juncture. The resulting loop transmission is shown in Fig. 3. Again, the only vehicle dynamics being considered are those of the rigid airframe.

(4) Employing a Simulink ${ }^{\circledR}$ simulation of the SMC system the existence of the sliding mode is verified. Figure 4 shows the inner-loop pitch-rate tracking performance and control signal for the pure SMC controller (no pilot or reference models). Figure 5 shows the corresponding 
pseudo-control signal $\mathrm{u}(\mathrm{t})$. Filtered white noise was used as the $\mathrm{q}_{\mathrm{d}}$ command signal. Still neglecting actuator dynamics, a $50 \%$ loss of stabilator and a thrust vector hard-over of $\delta_{1}=-3^{\circ}$ occurs at $25 \mathrm{sec}$. Note the invariance to parameter changes and the near infinite frequency switching in the pseudo-control signal. True infinite-frequency switching is not possible in simulation for the same reason that it is unachievable in practice.

(5) A boundary layer is now added and a minimum value of $\varepsilon=0.2$ is determined for the boundary layer width. Figures 6 and 7 show the same information as Figs. 4 and 5, except with the boundary layer included. The tracking performance with failure is degraded slightly from that possible with near-infinite switching frequency but now a continuous pseudo-control signal is in evidence.

(6) The actuators and structural bending mode dynamics are now included. Although not shown here, the controller fails to stabilize the vehicle with these parasitic dynamics.

(7) An asymptotic observer is created assuming the availability of noisy measurements of $\alpha(t)$ and $q(t)$. The eigenvalues of the observer are chosen as: $\lambda_{\text {obs }}=-10,-11$. These are seen to lie in the frequency range between the frequency limit and the bandwidth of the reference model. The resulting observer gain matrix $\mathbf{G}$ from Eqn. (8) is given in the appendix.

(8) Figure 8 shows the transfer function from the output of the reference model to the pitch rate of the vehicle, computed with the observer loop closed, and now including the actuator and flexible mode dynamics. Using a $-3 \mathrm{~dB}$ criterion, the bandwidth of this closed-loop system is $30 \mathrm{rad} / \mathrm{s}$, well below the $250 \mathrm{rad} / \mathrm{s}$ value obtained without the observer. This reduction corroborates the qualitative explanation of the rationale behind including an observer in the SMC design (Fig. 1). With the exception of the lightly damped structural mode, the minimum damping ratio of any of the complex eigenvalues of this linear closed-loop system is 0.53 . 
Equivalent Loop Transmissions Referring to Fig. 2, the loop transmission L of the unity feedback system that results in the same closed-loop transfer function $\left(\frac{q}{q_{d}}\right)$ as that shown in Fig. 8 can be obtained. This is given by:

$$
L=\frac{\left(q / q_{d}\right)}{1-\left(q / q_{d}\right)}
$$

Figure 9 shows the Bode plot of this transfer function where the structural mode has been omitted for clarity. Adequate gain and phase margins are in evidence. In addition, the $-30 \mathrm{~dB} / \mathrm{dec}$ slope of the magnitude portion of the Bode diagram around crossover corresponds exactly to the minimum value prescribed in classical loop-shaping designs. ${ }^{19}$ More representative measures of stability margins wherein feedback loops are broken at the actuators and sensors will be discussed in what follows. However, Fig. 9 illustrates an important point: Were one to attempt to design an equivalent controller for this vehicle using a series compensator in the forward loop, a crossover frequency of $19 \mathrm{rad} / \mathrm{sec}$ would be required. Given that the $q / \mathrm{u}$ vehicle dynamics exhibit a second-order roll-off and the actuators are of $2^{\text {nd }}$ order, second-order lead (two differentiations of an error signal) would be required of this compensator in a fairly broad frequency range around the $19 \mathrm{rad} / \mathrm{sec}$ crossover. This would result in serious sensor noise amplification at the actuator inputs as well as unfavorable interactions with any structural modes in the region. It is worthwhile to note that no signal differentiation is required in any of the elements of the SMC system of Fig. 2.

Figure 10 is a comparison of the Bode diagrams of the loop transmission defined in Eqn. (12) and that obtained using a compensator designed using loop-shaping techniques and given in the appendix. As the appendix indicates, the loop-shape design avoids the aforementioned $2^{\text {nd }}$ order lead, and consequently a crossover frequency of approximately $11 \mathrm{rad} / \mathrm{s}$ rather than 19 
$\mathrm{rad} / \mathrm{s}$ is now in evidence. The comparison of Fig. 10 suggests that the robust properties of the SMC controller can be interpreted in the frequency domain. Namely, these properties are attributable the SMC's ability to establish a relatively high crossover frequency while still maintaining significant phase and gain margins without the necessity of higher-order lead generation (error differentiation) occurring in the controller.

The role that the observer plays in permitting the higher crossover frequency in the SMC design can be appreciated by considering the loop transmission from Eqn. (12) in which the observer has been omitted. Figure 11 shows this loop transmission with negative stability margins clearly in evidence. Also shown is the loop transmission from Figs. 9 and 10 with observer eigenvalues $\lambda=-10,-11$. The role played by the observer is apparent in Fig. 11. With an observer, positive stability margins are obtained but at the cost of a reduced crossover frequency as compared to the system without the observer.

Robustness and Noise Amplification More traditional measures of robustness can be obtained by cutting the loops in Fig. 2 before each actuator and considering gain and phase margins, followed by a similar analysis in which each sensor loop is cut. It is obvious that such a stability analysis is not as rigorous as one obtained by inserting a perturbation matrix of the form

$$
P=\operatorname{diag}\left(K_{1} e^{-j \phi_{i}}, \ldots, K_{n} e^{-j \phi_{n}}\right)
$$

before the actuators or sensors and assessing closed-loop stability when $K_{i}$ and $\phi_{i}$ are varied within some desired region in the gain and phase parameter space. The approach used here was adopted for the sake of simplicity. Figure 12 shows the loop transmissions that result from such an analysis where actuator loops are cut with i referring to the loop in question. Ample stability margins are in evidence. Figure 13 shows similar results when sensor loops are cut. Finally, Fig. 14 shows the two closed loop transfer functions $u / n_{i}$ where $u$ is the pseudo-control and $n_{i}$ is a 
noise signal added to each sensor signal. Note the absence of roll-off in the noise amplification with increased frequency beyond the crossover frequency defined in Figs. 9 and 10. Roll-off does occur but only beyond the frequency range shown in Fig. 14. The largest noise amplification is $6.6 \mathrm{~dB}$ at $20 \mathrm{rad} / \mathrm{s}$ in the pitch-rate channel. This result probably constitutes the most serious weakness in the particular SMC design examined here. However, units are important at this juncture. Fig. 14 implies that an additive $20 \mathrm{rad} / \mathrm{s}$ sinusoidal noise signal with an amplitude $1 \mathrm{deg} / \mathrm{s}$ in the pitch-rate gyro (a significant amount of noise for such a device) would produce actuator commands with 2.14 deg RMS values.

\section{System Performance with Failures}

The pilot model for this vehicle and flight control system is created using the $\alpha$ frequency of $1.5 \mathrm{rad} / \mathrm{s}$ is used. A series of filtered step inputs with amplitudes of $\pm 10 \mathrm{deg}$ serves as the pitch-attitude command $\left(\theta_{c}\right)$.

The loop-shape control system design of the appendix is included for comparison purposes. The block diagram for this system is identical to that of Fig. 2 with the exception that no observer is used, and pitch-rate $q$ is the only feedback variable. The reference model and pilot model are identical to those for the SMC system. Sensor noise identical to that for the SMC system is used. The root-mean-squnare value of the sensor noise is relatively large. As a final constraint, an unmodeled $0.025 \mathrm{~s}$ time delay was included in the sensor loop(s) for both the SMC and loop-shape systems. Both the SMC and loop-shape systems were discretized using a Tustin transformation assuming a relatively low $20 \mathrm{~Hz}$ sampling rate. The manner in which the SMC system was discretized is shown in Fig. 15. The entire system including reference model was

replaced by a three-input $\left(\alpha, q, q_{c}\right)$, two-output $\left(\delta_{s c}, \delta_{t c}\right)$, discrete state-space representation. The loop-shape design was discretized in a similar manner, albeit as a two-input $\left(\mathrm{q}, \mathrm{q}_{\mathrm{c}}\right)$ two-output 
$\left(\delta_{\mathrm{sc}}, \delta_{\mathrm{lc}}\right)$, discrete state-space system. Each pilot/vehicle system was implemented in a Simulink ${ }^{\circledR}$ computer simulation the results of which will be described next.

Failure No. 1 The first simulated failure assumes a 50\% loss of stabililator area with a simultaneous failure of the thrust vectoring system (thrust vector frozen at $\delta_{\mathbf{t}}=-3 \mathrm{deg}$ ) and a 0.05 time delay introduced into the stabilator actuator. Figure 16 shows the pitch-attitude tracking performance of the pilot/vehicle SMC system when the failure is introduced $25 \mathrm{~s}$ into the run. As the figure shows, satisfactory tracking performance is retained after the failure. Figure 17 shows the stabilator time history. Also, the SMC design was insensitive to the characteristics of the approximate structural mode in that the order of the pole/zero combination could be reversed with no performance decrements. Finally, neither amplitude nor rate limits were met with either actuator before failure.

The loop-shape design is next subjected to the failure. Figures 18 and 19 show the pitchattitude tracking performance and stabilator time history. The tracking performance is considerably poorer than that of the SMC system in Fig. 16. In addition, the stabilator activity is larger for the loop-shape design after failure.

Failure No. 2 The second simulated airframe failure again assumes a 50\% loss of stabilator area. For the simulated actuator fąilures, both the thrust vectoring and stabilator actuators operate with a $0.05 \mathrm{~s}$ time delay and the stabilator actuator has its rate limits reduced from $\pm 60 \mathrm{deg} / \mathrm{s}$ to $\pm 10 \mathrm{deg} / \mathrm{s}$. Figure 20 shows the tracking performance of the pilot/vehicle SMC system when the failure is introduced $25 \mathrm{sec}$ into the simulation run. Figure 21 shows the stabilator actuator output rate. As Fig. 20 indicates, although some pitch overshoot is evident after failure (particularly around $\mathrm{t}=50 \mathrm{~s}$ ), the performance could be deemed acceptable, especially given the magnitude of the failures. Figure 21 indicates that after the failure, the stabilator actuator is in almost constant rate saturation. This performance is possible here since $\sigma$ 
always remains within the boundary layer. Unfortunately, this condition cannot be guaranteed with SMC systems if rate or amplitude limits are reached with all effectors. Methods for explicitly handling amplitude and rate saturation require knowledge of both the actuator dynamics and their saturation characteristics, conditions not likely to be met when damage occurs. $^{21}$ The robust performance shown in Fig. 20 is attributable to the SMC system being implemented with two control effectors, one of which (the thrust vector control) did not have reduced saturation characteristics in the assumed failure. As with the first failure, the SMC design was insensitive to the characteristics of the approximate structural mode.

The loop shape system is next subjected to an identical failure. Figure 22 shows the pitchattitude tracking performance. As can be seen, the system undergoes an oscillatory instability soon after the introduction of the failure.

\section{Discussion}

The issue of bandwidth deserves some comment at this juncture. By their nature, SMC designs tend to be high-bandwidth systems. This has been emphasized in the preceding sections. The problems that can accompany high-bandwidth flight control system designs are well known ${ }^{22}$. These problems include (1) insufficient stability margins due to unmodeled (parasitic) dynamics, (2) the excitation of unmodeled high-frequency modes, such as those associated with the vehicle structure, (3) actuator saturation, or at best, unduly high control activity, (4) high sampling rates in digital control law implementation, and (5) sensor noise amplification at the actuator input. The authors have attempted to address each of these issues through an example, albeit one which utilizes a very simple vehicle aerodynamic model. While certainly not immune to the problems just enumerated, the SMC design approach that has been presented appears to render them manageable. The robustness benefits that appear to accrue may justify the additional attention that needs to be paid to bandwidth-related issues. 
There are no intrinsic limitations of the proposed methodology to SISO systems. This is demonstrated in Ref. 23. Under the assumption of a square system, where the number of reference model inputs are equal to the outputs to be feedback to the SMC system, the design of robust, decoupled controllers is reasonably straightforward. The methodology proposed here for SISO designs can be applied independently to each of the control channels of the MIMO system. Again, frequency domain techniques could be employed to assess the quality of the resulting linear design.

The SMC design approach discussed here has also been successfully applied to the design of a longitudinal flight control system for a model of a forward swept-wing fighter aircraft. ${ }^{24}$ The model included two structural modes consisting of wing bending and torsion with modal characteristics obtained from finite element analysis. The vehicle model is also highly unstable with a divergent mode exhibiting a time to double amplitude of less than $0.1 \mathrm{~s}$.

\section{Conclusions}

A step-by-step procedure for the design of sliding mode controllers for reconfigurable flight control systems is feasible. Asymptotic observers have been shown to eliminate the well-documented sensitivity of sliding mode designs to parasitic dynamics. The procedure allows a number of the required design decisions to be made in the frequency domain, and allows an interpretation of the robust properties of the SMC controller in terms of an effective, unity-feedback loop transmission. Viewed in this light, the SMC procedure serves as a means to an end, that being the creation of a loop transmission with significant stability and performance robustness in the presence of system failures.

In a computer simulation of a fighter pitch-rate flight control system including a model of the human pilot, the SMC system exhibits tracking and robustness superior to that exhibited by a 
system designed using classical, loop-shaping techniques. This comparison included significant airframe and actuator damage with the comparison undertaken with discretized controllers.

The absence of requirements for failure detection, system identification, and on-line algorithm redesign makes the SMC approach attractive from the standpoint of vehicle handling qualities. The most serious weakness in the present SMC application is the lack of magnitude roll-off in the transfer function between actuator input and sensor noise at frequencies beyond the closed-loop system bandwidth. However, the noise amplification at these frequencies is considerably less than that which would be produced by a classical loop-shaping design with the same bandwidth as the SMC system.

\section{Acknowledgement}

This research was supported by a grant from NASA Langley Research Center. Dr. Barton Bacon serves as the grant technical manager.

\section{Appendix}

Nominal and Damaged Vehicle, and Sensor Noise Models

The nominal flight condition is a Mach number of 0.6 at an altitude of $30,000 \mathrm{ft}$.

$$
\dot{\mathbf{x}}(t)=\mathbf{A x}(t)+B u(t) \quad \mathbf{x}(t)=[\alpha(t) q(t)] \quad u(t)=\left[\delta_{s} \delta_{1}\right]
$$

where $\alpha$ is angle of attack, deg; $q$ is pitch rate, deg/sec; $\delta_{\mathrm{s}}$ is stabilator angle, deg; and $\delta_{\mathrm{t}}$ is thrust vector angle, deg. For the nominal vehicle,

$$
\mathbf{A}=\left[\begin{array}{cc}
-0.5088 & 0.994 \\
-1.131 & -0.2804
\end{array}\right] \quad \mathbf{B}=\left[\begin{array}{cc}
-0.9277 & -0.01787 \\
-6.575 & -1.525
\end{array}\right]
$$

for the damaged vehicle ( $50 \%$ reduction in stabilator area)

$$
\mathbf{A}_{\text {fail }}=\left[\begin{array}{cc}
-0.48 & 0.997 \\
-0.4639 & -0.01787
\end{array}\right] \quad \mathbf{B}_{\text {fail }}=\left[\begin{array}{cc}
-0.4639 & -0.01787 \\
-3.2875 & -1.525
\end{array}\right]
$$

The observer gain matrix $\mathbf{G}$ yielding eigenvalues of $\lambda_{\text {obs }}=-10,-11$ is given by 


$$
G=\left[\begin{array}{cc}
9.4912 & 0.994 \\
-1.131 & 10.7196
\end{array}\right]
$$

To approximate the effects of a flexible body-bending mode on the aircraft, the $\alpha$ and $q$ outputs were passed through a second order dipole given by $\left[60^{2} / 50^{2}\right]\left(\mathrm{s}^{2}+2(0.005)(50) \mathrm{s}+50^{2}\right) /\left(\mathrm{s}^{2}+2(0.005)(60) \mathrm{s}+60^{2}\right)$. The actuator model for the stabilator is $30^{2} /\left(\mathrm{s}^{2}+42.4 \mathrm{~s}+30^{2}\right)$, with amplitude limits of $\pm 30 \mathrm{deg}$, and rate limits of \pm 60 $\mathrm{deg} / \mathrm{s}$. The actuator model for the thrust vectoring is $20^{2} /\left(\mathrm{s}^{2}+24 \mathrm{~s}+20^{2}\right)$, with amplitude limits of $\pm 30 \mathrm{deg}$, and rate limits of $\pm 60 \mathrm{deg} / \mathrm{s}$. Sensor noise in the $\alpha$ and $\mathrm{q}$ sensors are modeled as white noise passed through filters $10^{2} /\left(\mathrm{s}^{2}+20 \mathrm{~s}+10^{2}\right)$ producing RMS noise levels of $0.5 \mathrm{deg}$, and $0.5 \mathrm{deg} / \mathrm{s}$, respectively. The pilot model for both the SMC and loop-shape designs is given in shorthand notation by

$$
Y_{p}=\frac{1.5 \cdot 10^{5}(5) e^{-0.2 s}}{[0.15,8.69](16.75)[0.67,25.9]}
$$

The model includes the dynamics of a second-order force/feel system with transfer function with undamped natural frequency of $25 \mathrm{rad} / \mathrm{s}$ and damping ratio 0.707 . The loop-shape compensator is given by

$$
\mathrm{G}_{\text {loop }}=\frac{-1.24[0.365,1.1]}{(0)(0.3)}
$$




\section{References}

'Itkis, Y., Control Systems of Variable Structure, Wiley, New York, NY, 1976.

${ }^{2}$ Utkin, V. I., "Variable Structure Systems with Sliding Mode," IEEE Transactions on Automatic Control, Vol. AC-22, No. 2, 1977, pp. 212-222.

${ }^{3}$ Utkin, V. I., Sliding Modes in Control and Optimization, Springer-Verlag, Berlin, Ger., 1992.

${ }^{4}$ Edwards, C., and Spurgeon, S. K., Sliding Mode Control, Taylor \& Francis Ltd, Bristol, PA, 1998.

${ }^{5}$ Hung, J. Y., Weibing, G., and Hung, J. C., "Variable Structure Control: A Survey," IEEE Transactions on Industrial Electronics, Vol. 40, No. 1, 1993, pp. 2-23.

${ }^{6}$ Poznyak, A. S., Yu, W., and Sanchez, E. N., "Identification and Control of Unknown Chaotic Systems via Dynamic Neural Networks," IEEE Transactions on Circuits and Systems-I: Fundamental Theory and Applications, Vol. 46, No. 12, 1999, pp. 1491-1495.

${ }^{7}$ Levant, A., "Robust Exact Differentiation via Sliding Mode Technique," Automatica, Vol. 34, No. 3, 1998, pp. 379-384.

${ }^{8}$ Salamci, M. U., Özgören, M. K., and Banks, S. P., "Sliding Mode Control with Optimal Sliding Surfaces for Missile Autopilò Design," Journal of Guidance, Control, and Dynamics, Vol. 23, No. 4, 2000, pp. 719-727.

${ }^{9}$ Schumacher, C., and Singh, S. N., "Nonlinear Control of Multiple UAVs in CloseCoupled Formation Flight," AIAA-2000-4373, Proceedings of Guidance, Navigation, and Control Conference, AIAA, Denver, CO, 14-17 Aug 2000, pp. 1-7.

${ }^{10}$ Shtessel, Y. B., Buffington, J. M., and Banda, S. S., "Multiple Timescale Flight Control Using Reconfigurable Sliding Modes," Journal of Guidance, Control, and Dynamics, Vol. 22, No. 6, 1999, pp. 873-883. 
${ }^{11}$ DeCarlo, R., A., Zak, S. H., and Mathews, G. P., "Variable Structure Control of Nonlinear Multivariable Systems: A Tutorial," Proceedings of the IEEE, Vol. 76, No. 3, 1988, pp. 212-232.

${ }^{12}$ DeCarlo, R., A., Zak, S. H., and Drakunov, S. V., "Section 57.5: Variable Structure, Sliding Mode Design," The Control Handbook, Levine, W. S. eds., CRC Press, Inc., Boca Raton, FL, 1996, pp. 941-951.

${ }^{13}$ Fernández, B. R., and Hedrick, J. K., "Control of Multivariable Non-Linear Systems by the Sliding Mode Method," International Journal of Control, Vol. 46, No. 3, 1987, pp. 10191040.

${ }^{14}$ Utkin, V. I., "Sliding Mode Control Design Principles and Applications to Electric Drives," IEEE Transactions on Industrial Electronics, Vol. 40, No. 1, 1993, pp. 23-36.

${ }^{15}$ Young, K. D., Utkin, V. I., and Özgüner, Ü., "A Control Engineer's Guide to Sliding Mode Control," IEEE Transactions on Control Systems Technology, Vol. 7, No. 3, 1999, pp. 328-342.

${ }^{16}$ Slotine, J.-J. E., and Li, W., Applied Nonlinear Control, Prentice Hall, Englewood Cliffs, NJ, 1991.

${ }^{17}$ Siwakosit, W., Snell, S. A.; and Hess, R. A., "Robust Flight Control Design with Handling Qualities Constraints Using Scheduled Linear Dynamic Inversion and Loop-Shaping," IEEE Transactions on Control Systems Technology, Vol. 8, No. 3, 2000, pp. 483-494.

${ }^{18}$ Hess, R. A., "Unified Theory for Aircraft Handling Qualities and Adverse Aircraft-Pilot Coupling," Journal of Guidance, Control, and Dynamics, Vol. 20, No. 6, 1997, pp. 1141-1148.

${ }^{19}$ Maciejowski, J. M., Multivariable Feedback Design, Addison-Wesley, Wokingham, UK, 1989, Chap. 1. 
${ }^{20}$ Adams, R. J., Buffington, J. M., Sparks, A. G., and Banda, S. S., "An Introduction to Multivariable Flight Control Design," USAF Wright Laboratory, WL-TR-92-3110, WrightPatterson AFB, OH, Oct, 1992.

${ }^{21}$ Shtessel, Y. B., Buffington, J. M., Pachter, M., Chandler, P.R., and Banda, S., S., "Reconfigurable Flight Control on Sliding Modes Addressing Actuator Deflections and Deflection Rate Saturation, AIAA Paper No. 98-4112.

${ }^{22}$ McRuer,D., Ashkenas, I., and Graham, D., Aircraft Dynamics and Automatic Control, Princeton University Press, Princeton, NJ, 1973, Chap. 9.

${ }^{23}$ Wells, S. R. and Hess, R. A., "MIMO Sliding Mode Control for a Tailless Fighter Aircraft - An Alternative to Reconfigurable Architectures," AIAA Paper No. 2002-4650.

${ }^{24}$ Vetter, T. K., "Sliding-Mode Control Applied for Robust Control of a Highly Unstable Aircraft,' MS Thesis, 2002, Dept. of Mechanical and Aeronautical Engineering, University of California, Davis CA. 


\section{Figure Captions}

Figure $1 \quad$ Asymptotic observers in SMC systems

Figure 2 SMC pilot/vehicle system

Figure 3 Loop transmission for SMC design, no actuators, flexible mode or observer included

Figure 4 SMC tracking performance with sliding mode and failure. No actuator dynamics or observer included

Figure 5 SMC pseudo-control input with sliding mode showing near-infinite frequency switching

Figure 6 SMC tracking performance with boundary layer and failure. No actuator dynamics or observer included

Figure 7 SMC pseudo-control input with boundary layer showing continuous control

Figure 8 Closed loop SMC/vehicle transfer function from reference model output to vehicle pitch rate; actuators, flexible mode and observer included

Figure 9 Effective unity feedback loop transmission for SMC design; flexible mode removed for clarity

Figure 10 Comparison of loop transmission from SMC and loop-shape designs

Figure $11 \quad$ Loop transmission for SMC design with and without observer

Figure 12 SMC loop transmission with loop cut before $i^{\text {th }}$ actuator; $i=1$ refers to stabilator, $\mathrm{i}=2$ refers to thrust vectoring

Figure 13 SMC loop transmission with loop cut at $i^{\text {th }}$ sensor; $i=1$ refers to $\alpha$ sensor, $i=2$ refers to q sensor

Figure 14 Sensor noise amplification, $\mathrm{u} / \mathrm{n}_{\mathrm{i}}, \mathrm{n}_{1}$ refers to noise added to $\alpha$ signal, $\mathrm{n}_{2}$ refers to noise added to q signal

Figure 15 Descretization of SMC system

Figure 16 SMC pitch-attitude tracking before and after Failure No. 1

Figure 17 SMC stabilator time history before and after Failure No. 1

Figure 18 Loop-shape system pitch-attitude tracking before and after Failure No. 1

Figure 19 Loop-shape system stabilator time history before and after Failure No. 1

Figure 20 SMC pitch-attitude tracking before and after Failure No. 2

Figure 21 SMC stabilator-rate time history before and after Failure No. 2

Figure 22 Loop-shape system pitch-attitude tracking before and after Failure No. 2 


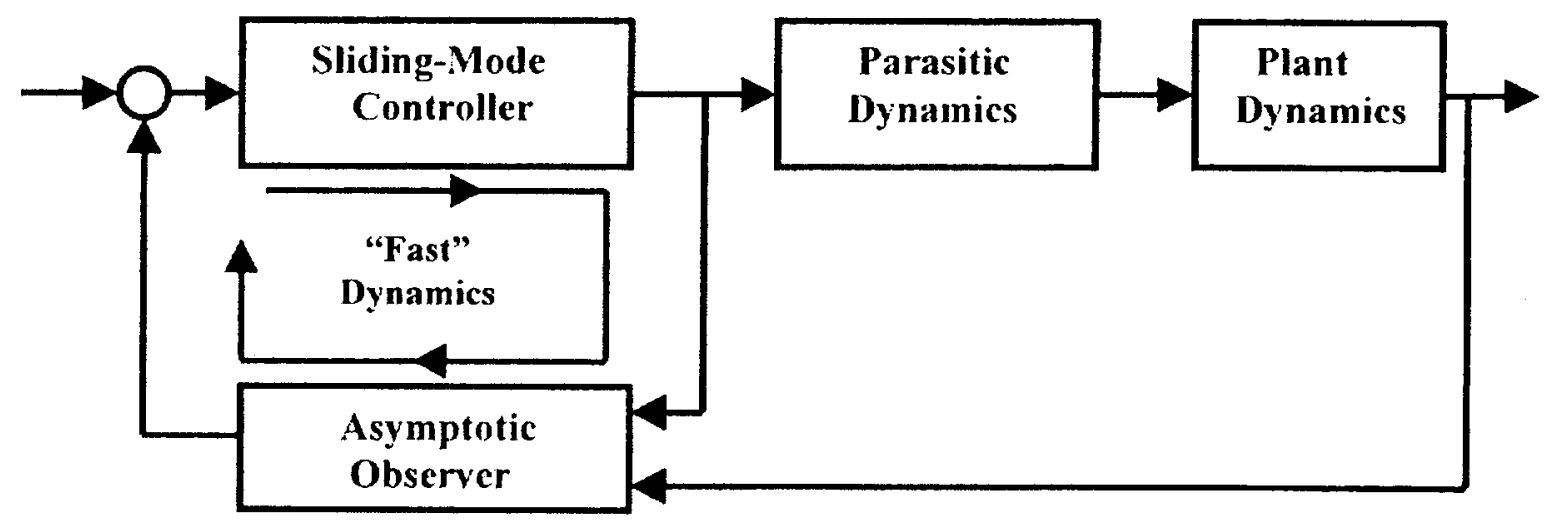

Figure 1 Asymptotic observers in SMC systems 


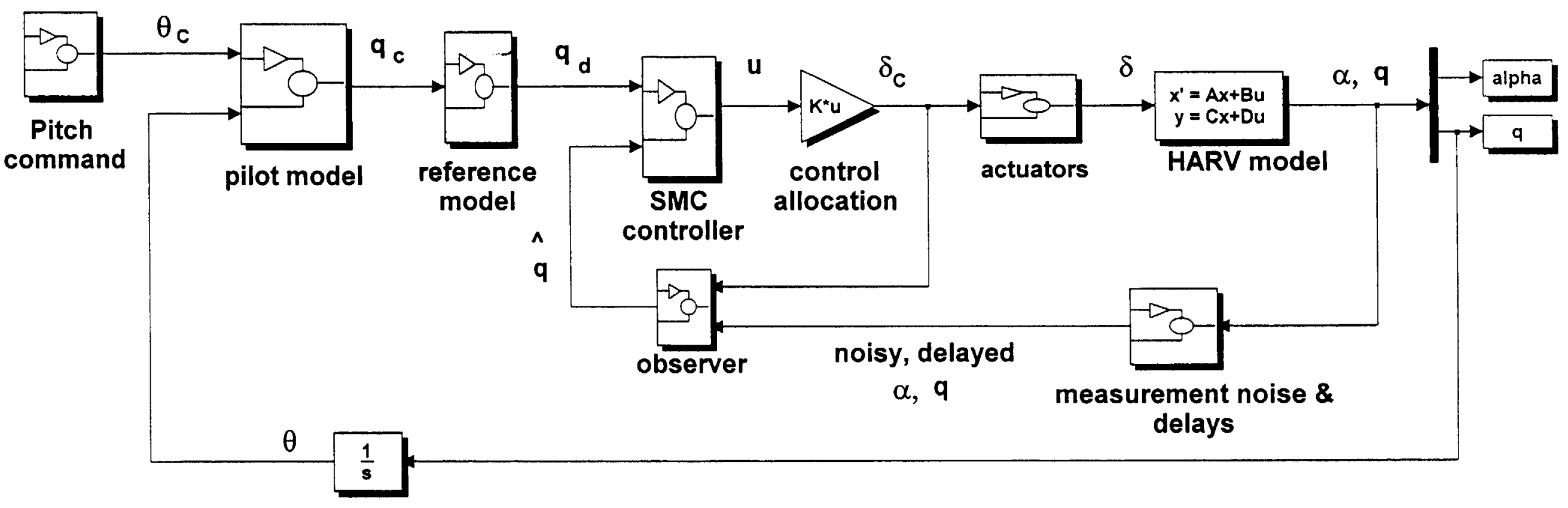

Figure 2 SMC pilot/vehicle system 

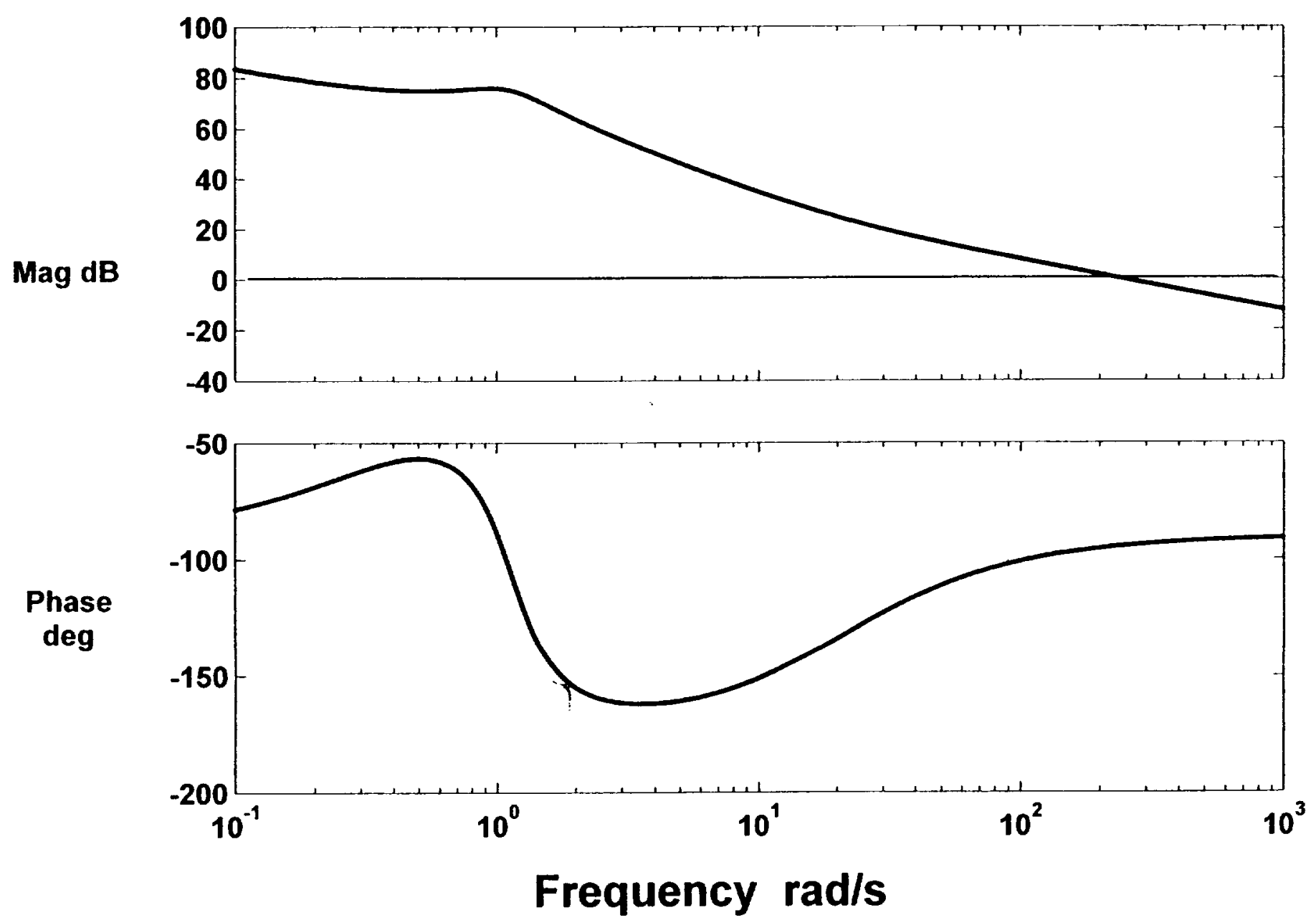

Figure 3 Loop transmission for SMC design, no actuators, flexible mode or observer included 


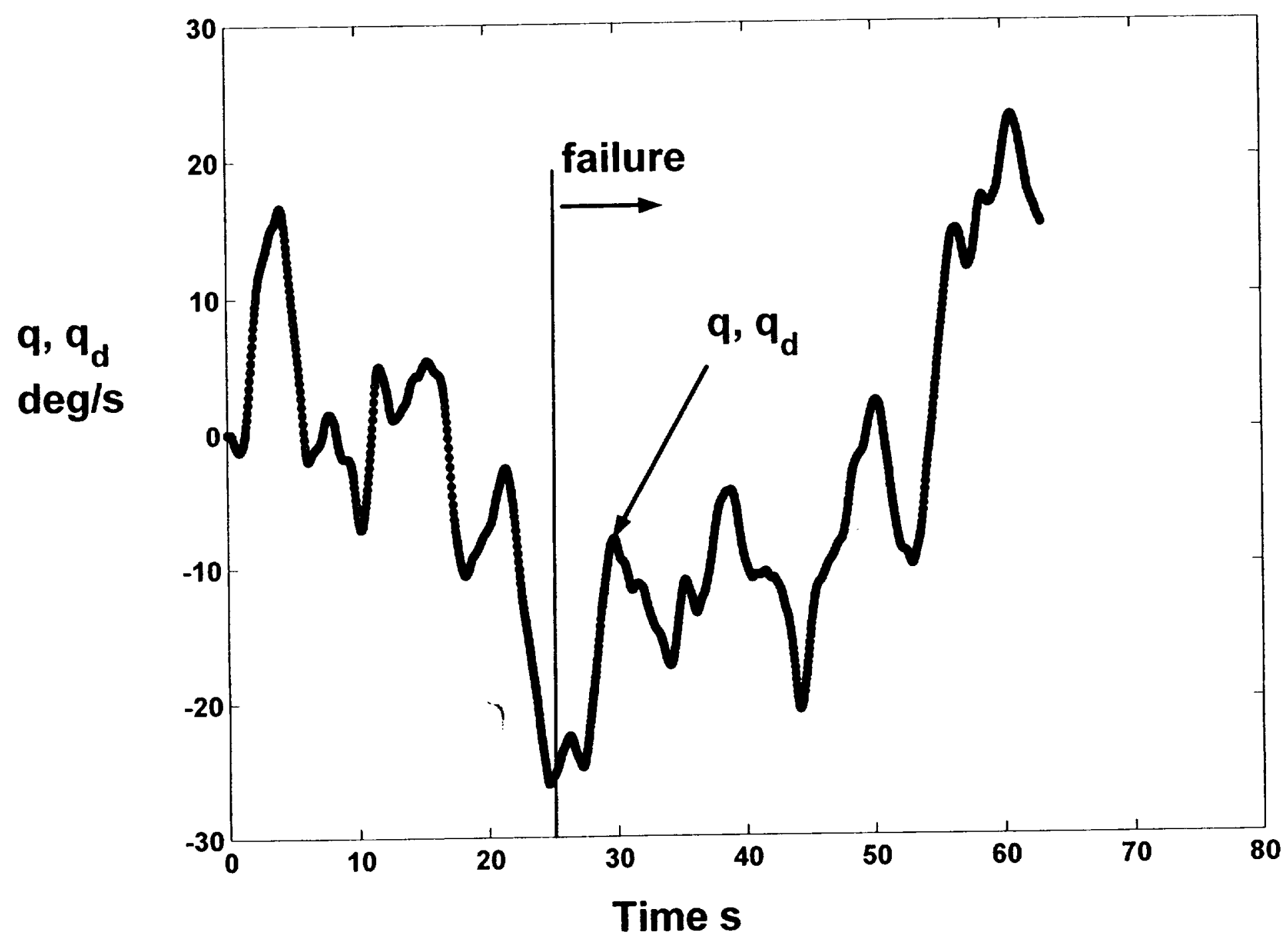

Figure 4 SMC tracking performance with sliding mode and failure. No actuator dynamics or observer included 


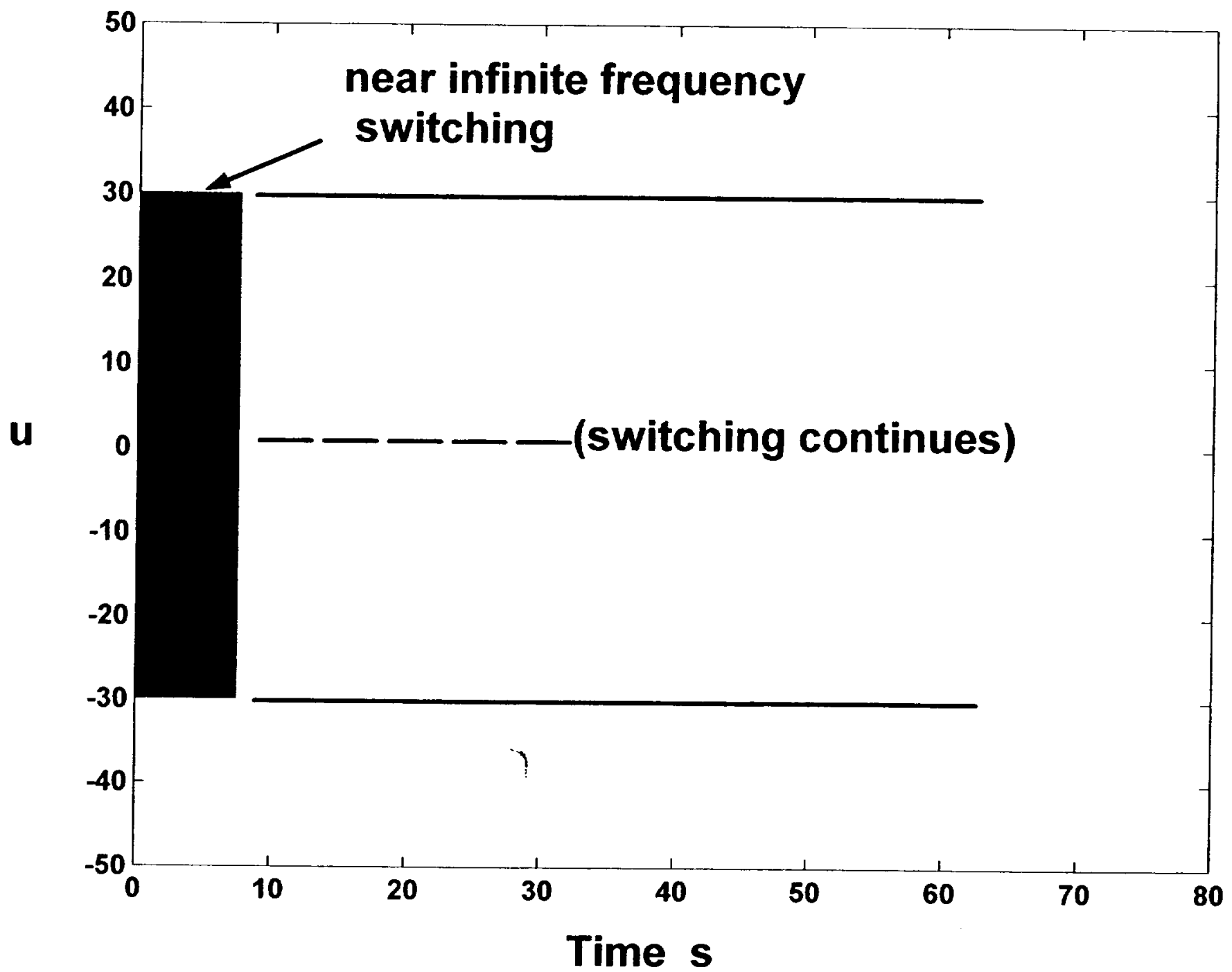

Figure 5 SMC pseudo-control input with sliding mode showing near-infinite frequency switching 


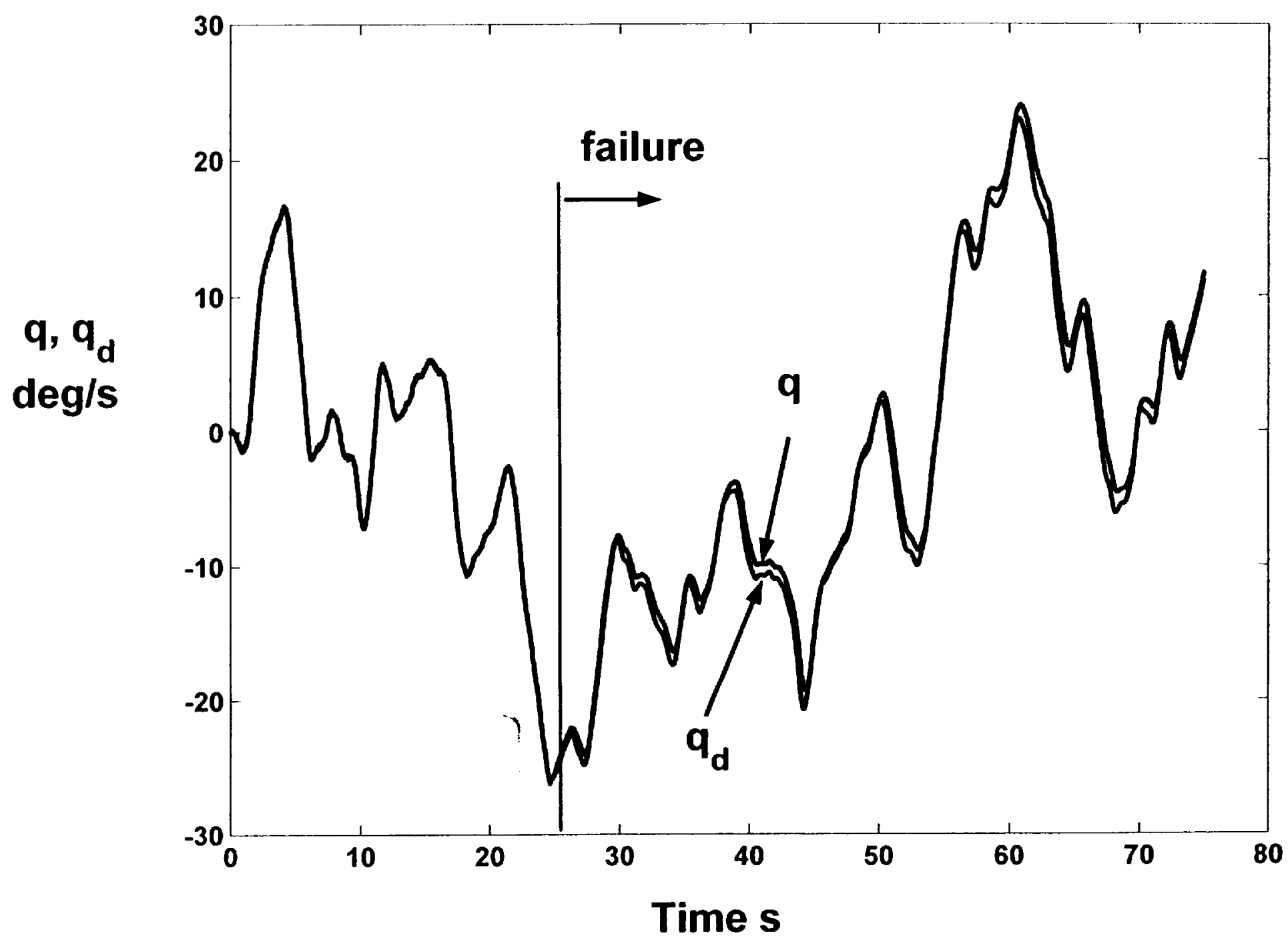

Figure 6 SMC tracking performance with boundary layer and failure. No actuator dynamics or observer included 


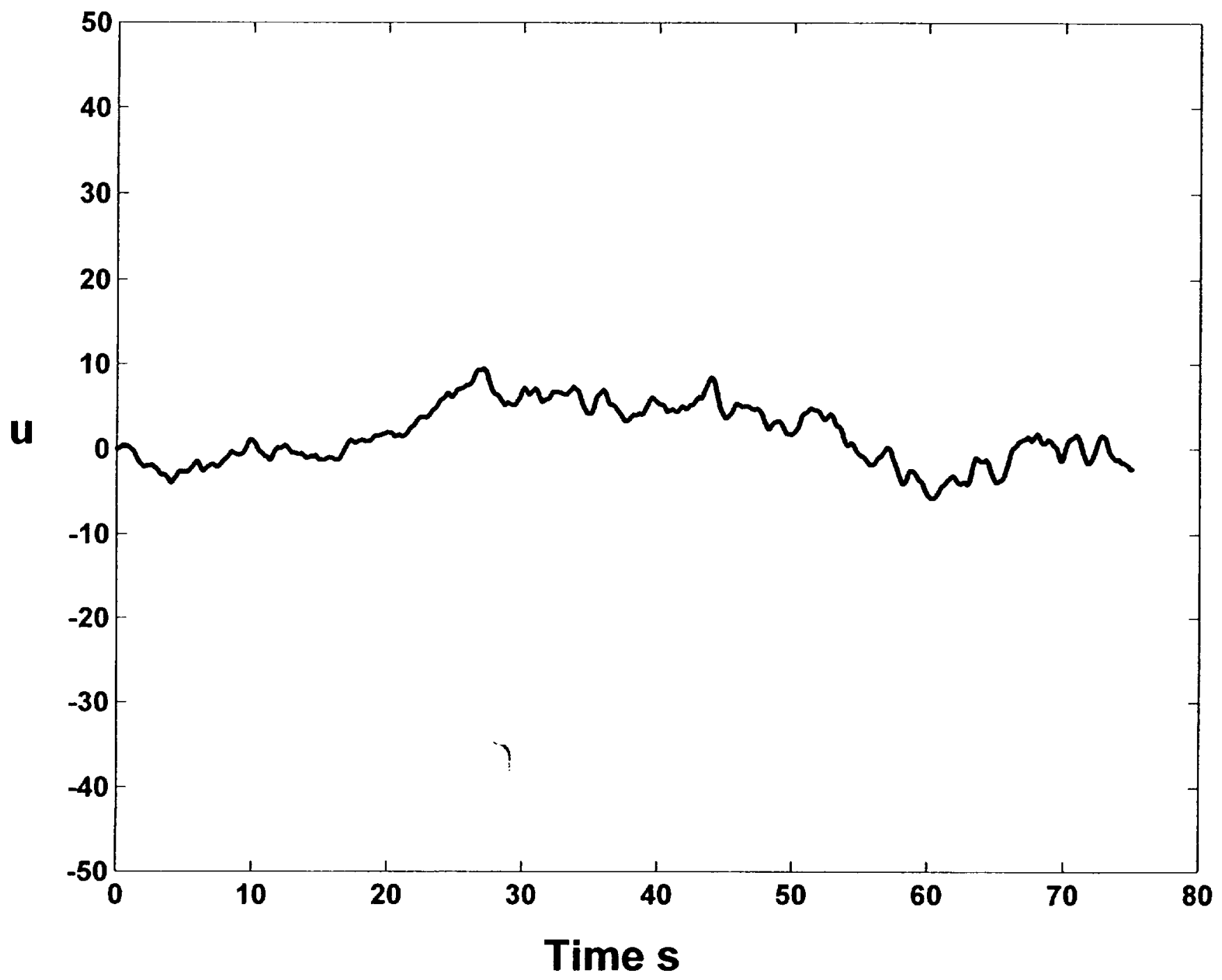

Figure 7 SMC pseudo-control input with boundary layer showing continuous control 


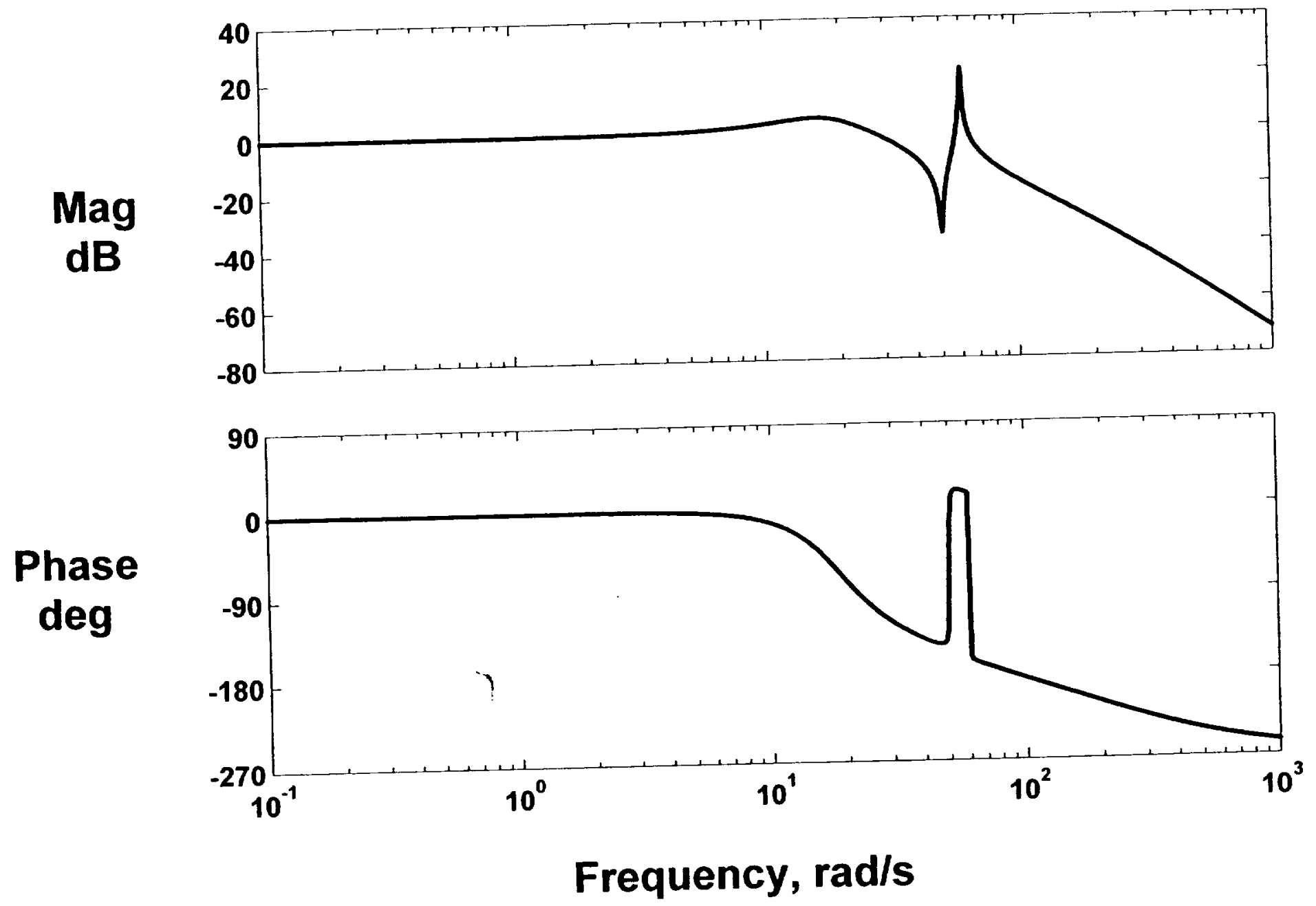

Figure 8 Closed loop SMC/vehicle transfer function from reference model output to vehicle pitch rate; actuators, flexible mode and observer included 


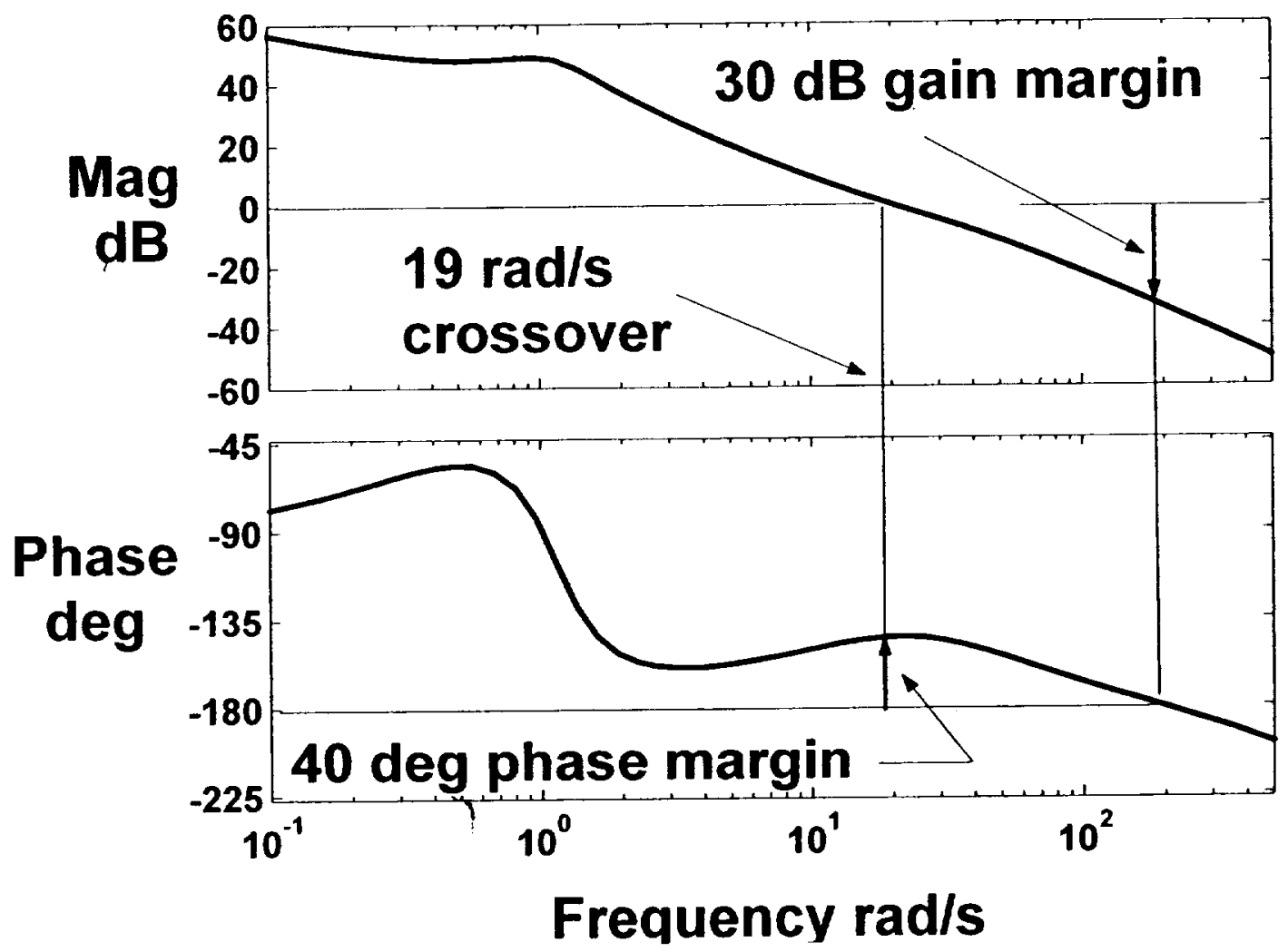

Figure 9 Effective unity feedback loop transmission for SMC design; flexible mode removed for clarity 


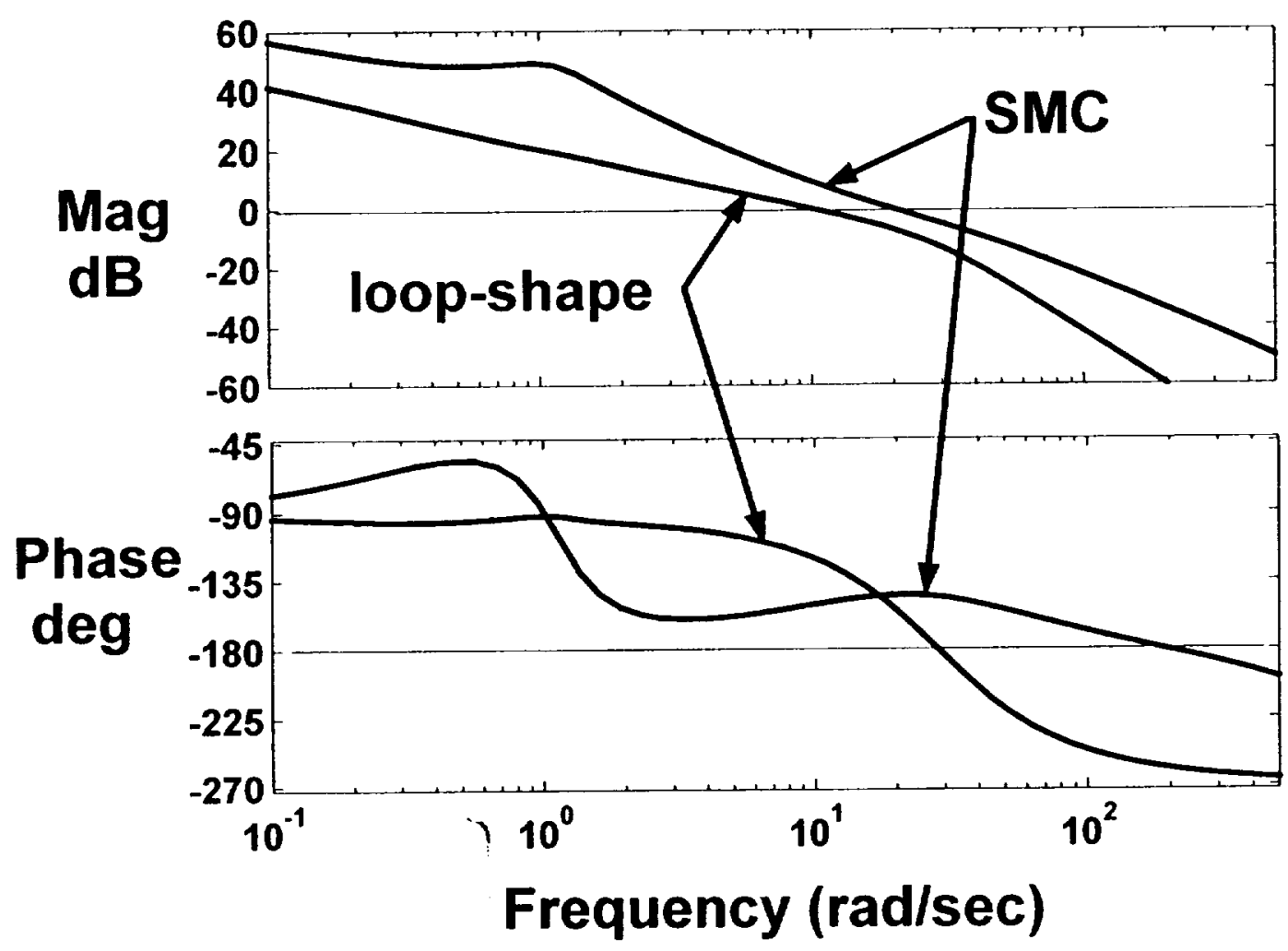

Figure 10 Comparison of loop transmission from SMC and loop-shape designs 


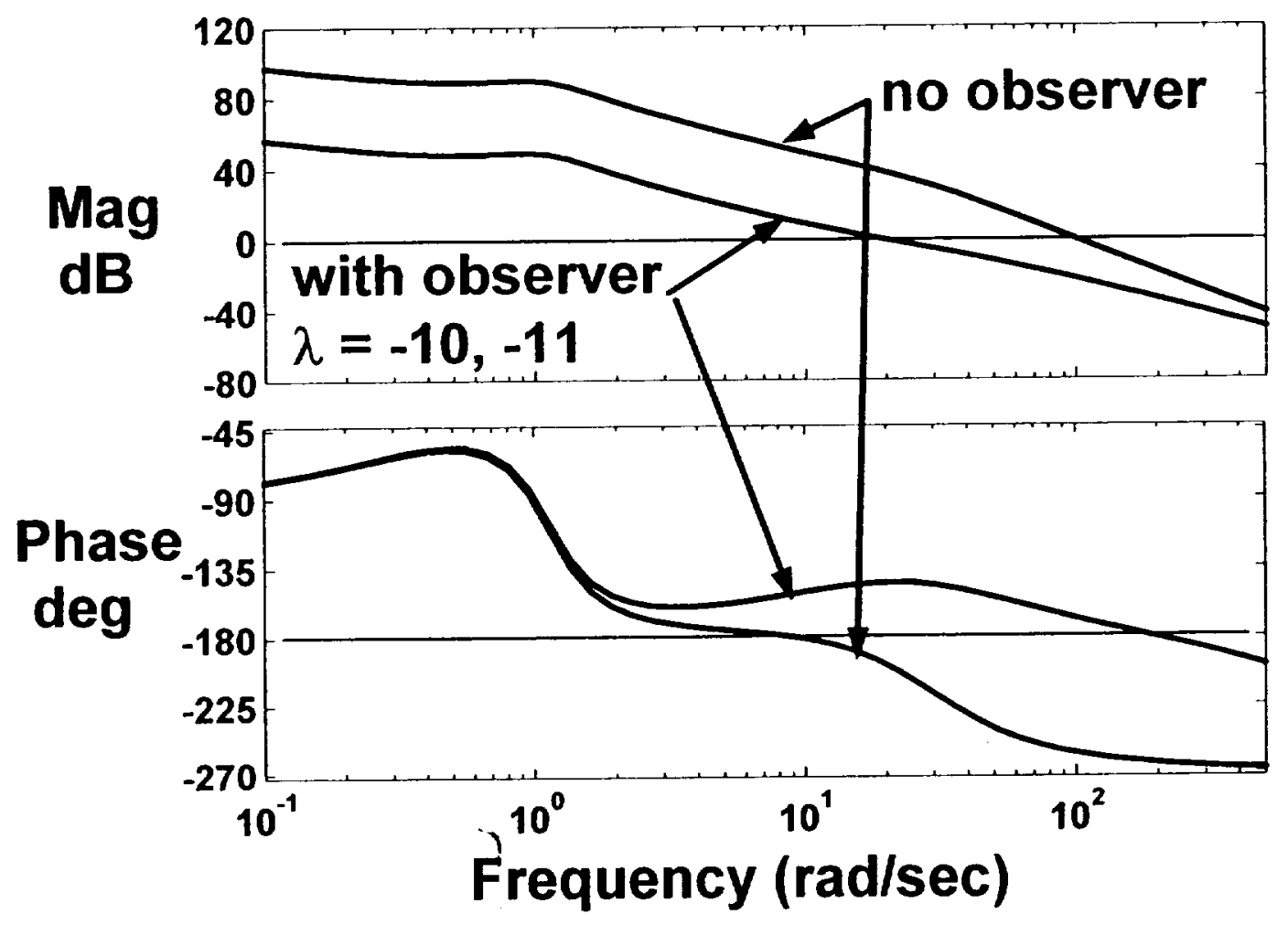

Figure 11 Loop transmission for SMC design with and without observer 


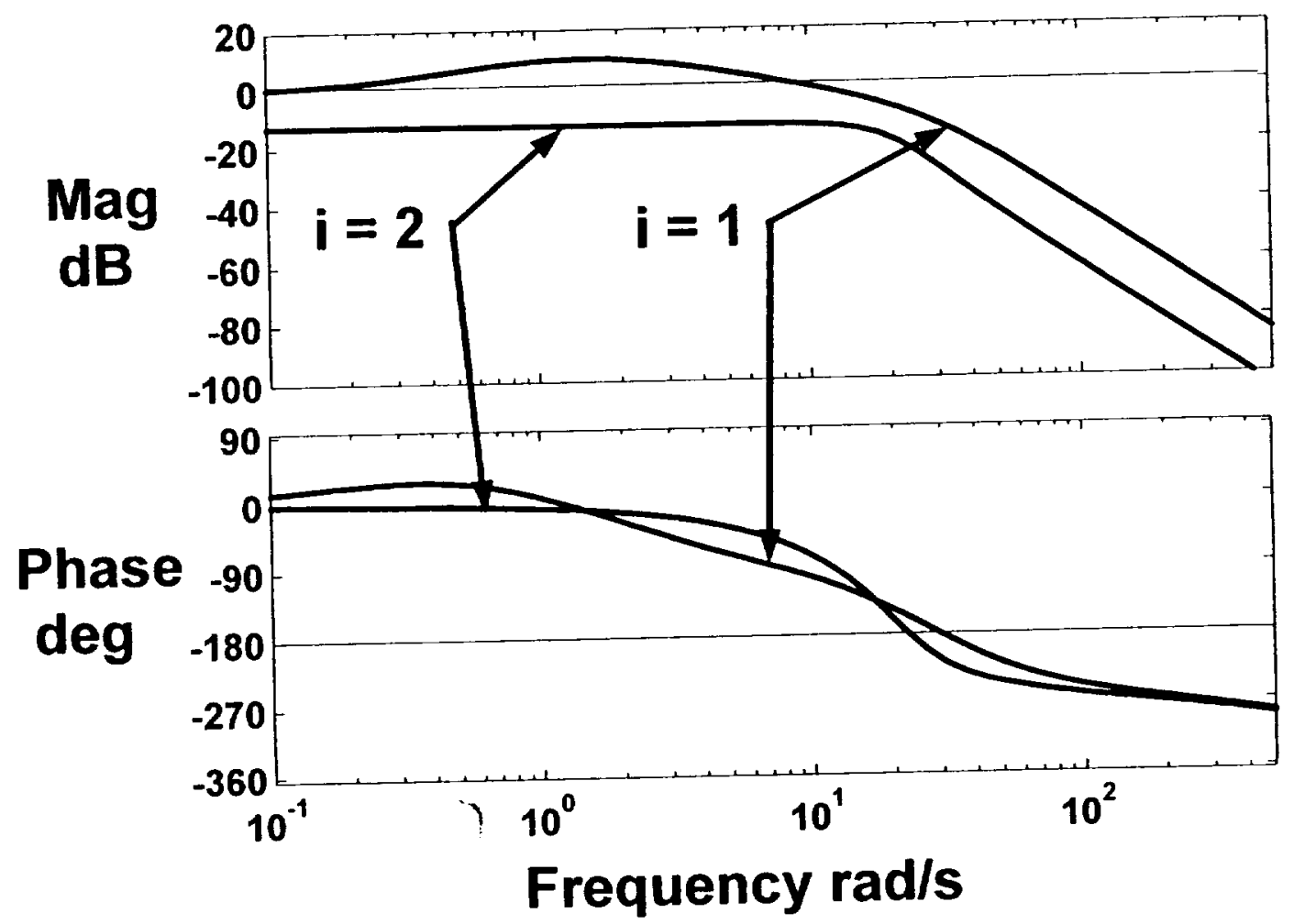

Figure 12 SMC loop transmission with loop cut before $i^{\text {th }}$ actuator; $i=1$ refers to stabilator, $i=2$ refers to thrust vectoring 


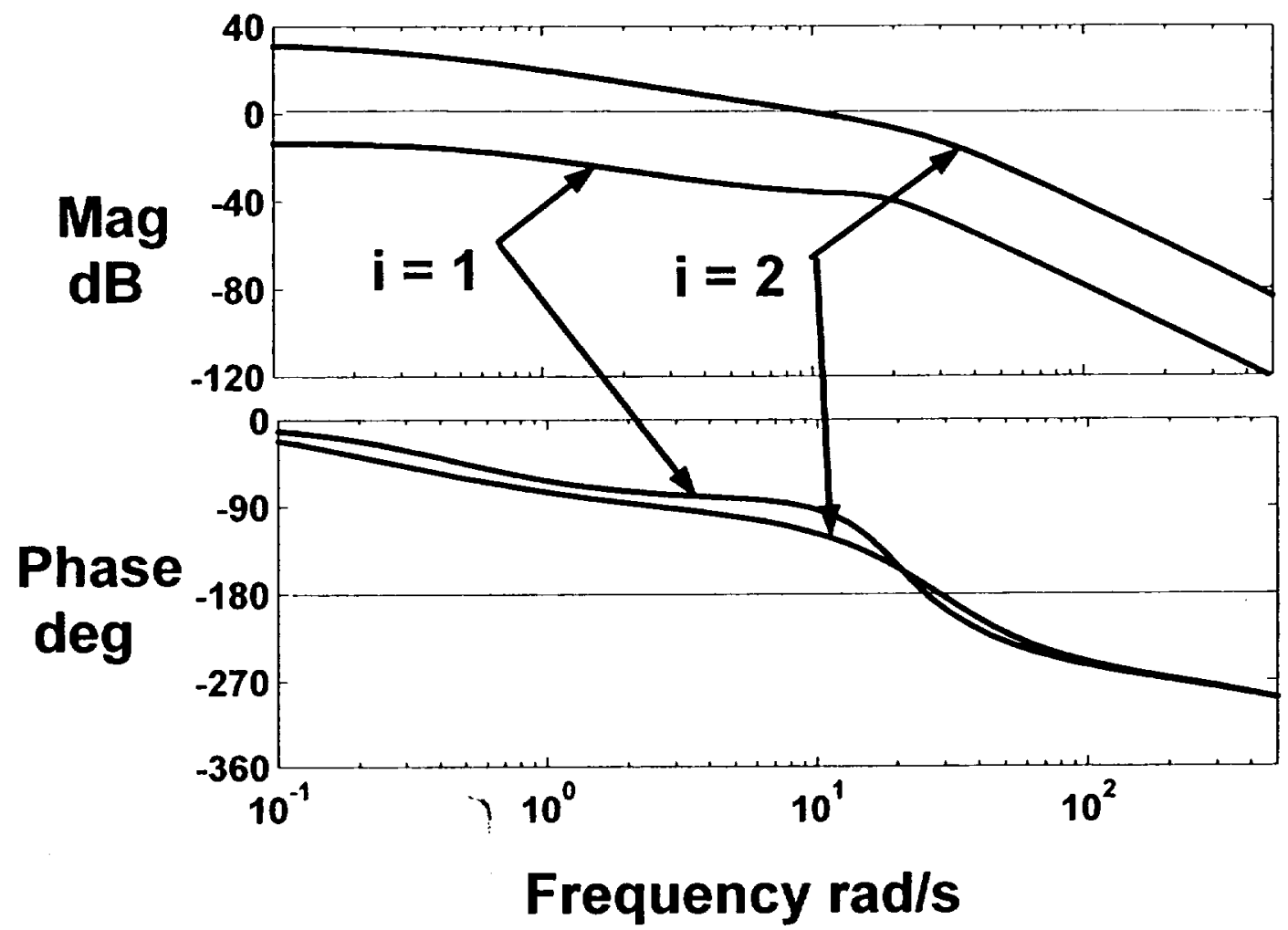

Figure 13 SMC loop transmission with loop cut at $i^{\text {th }}$ sensor; $i=1$ refers to $\alpha$ sensor, $i=2$ refers to q sensor 


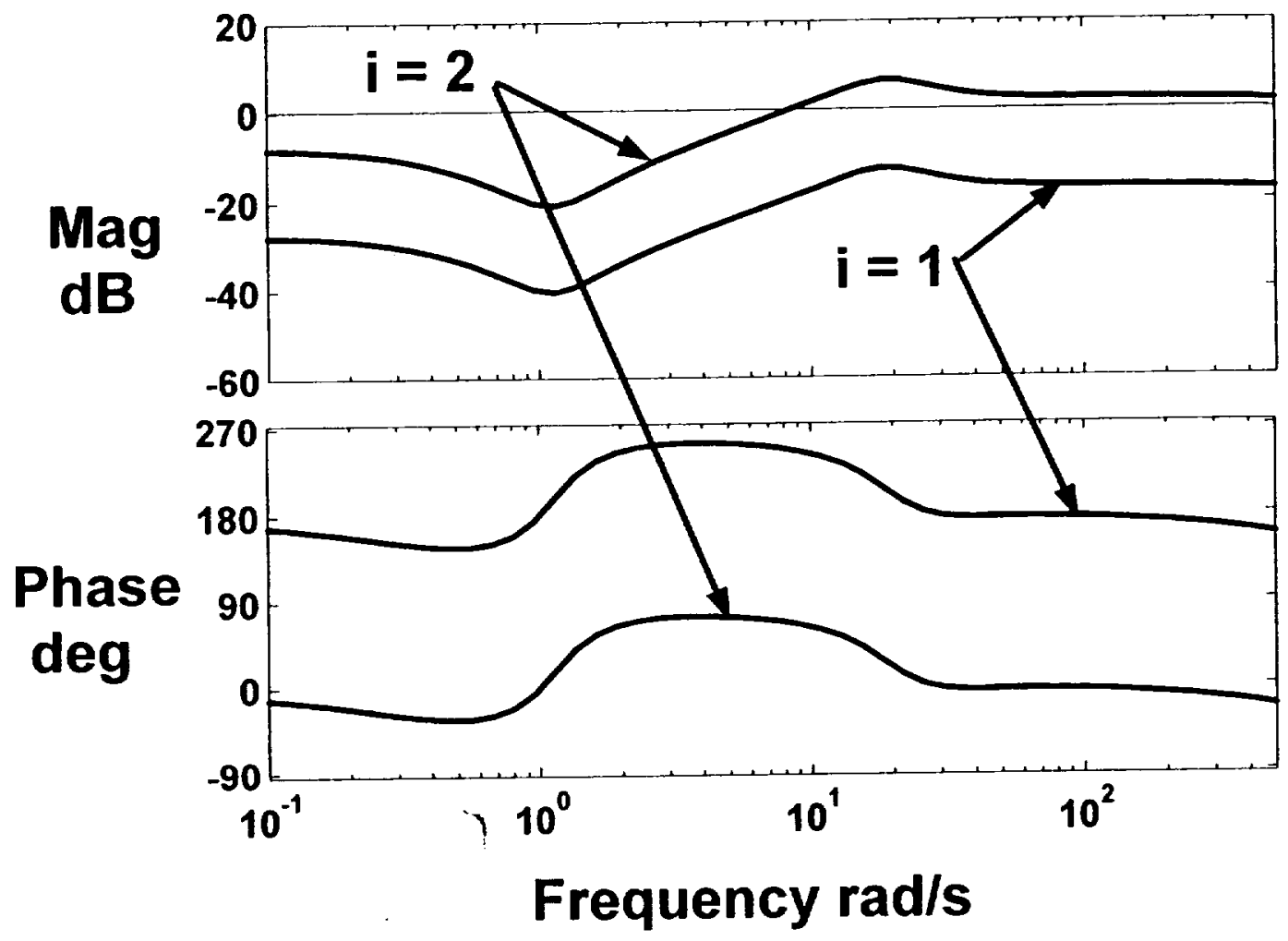

Figure 14 Sensor noise amplification, $\mathrm{u} / \mathrm{n}_{\mathrm{i}}, \mathrm{n}_{\mathbf{l}}$ refers to noise added to $\alpha$ signal, $\mathrm{n}_{2}$ refers to noise added to $\mathrm{q}$ signal 


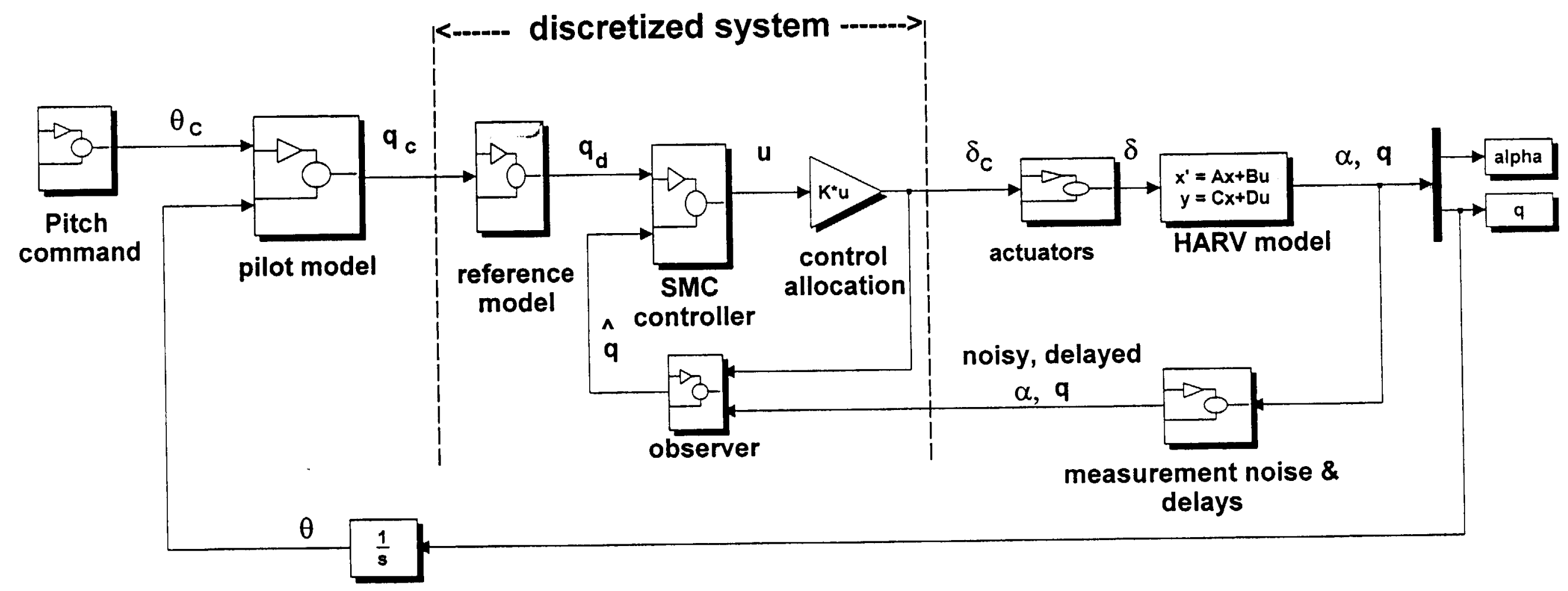

Figure 15 Descretization of SMC system 


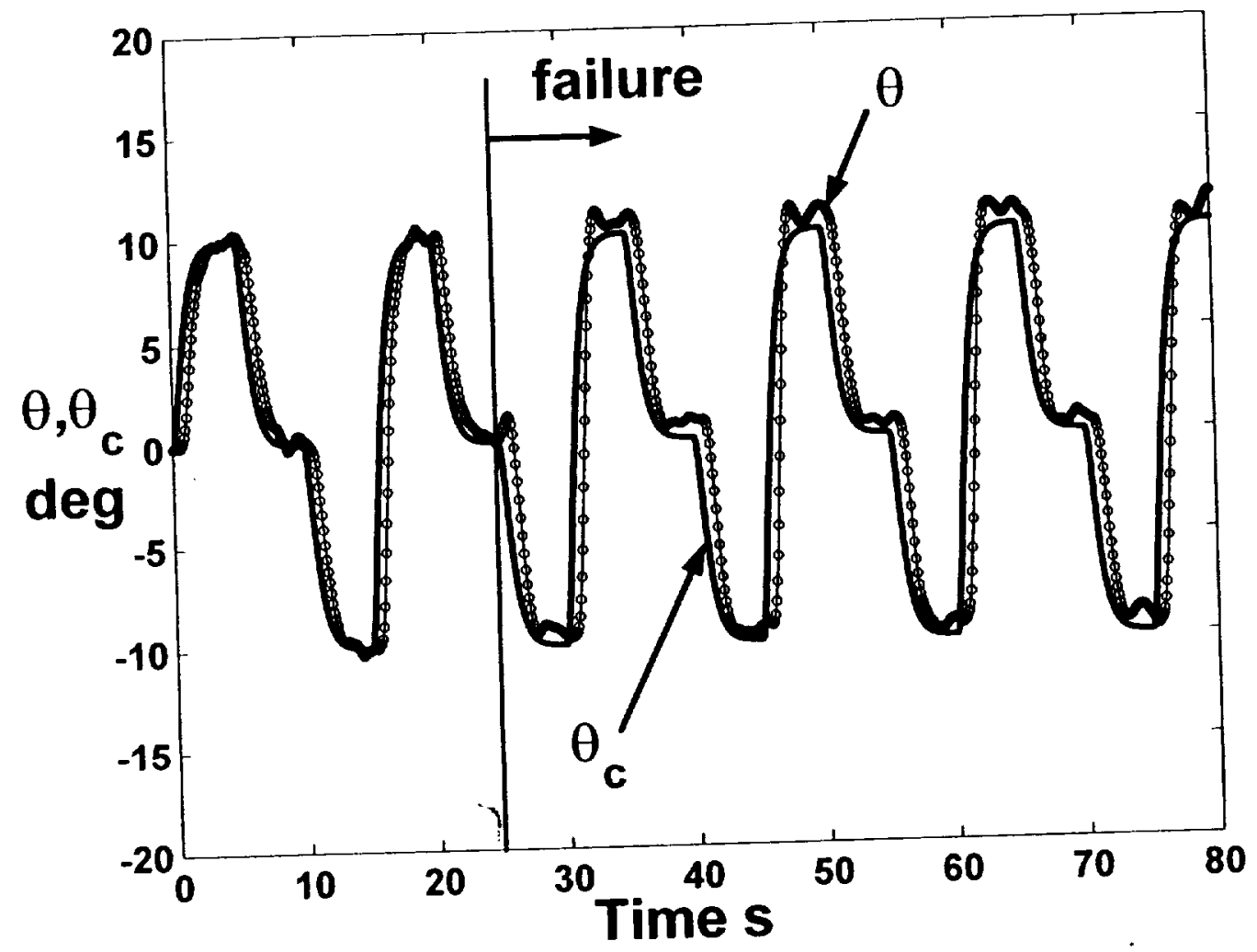

Figure 16 SMC pitch-attitude tracking before and after Failure No. 1 


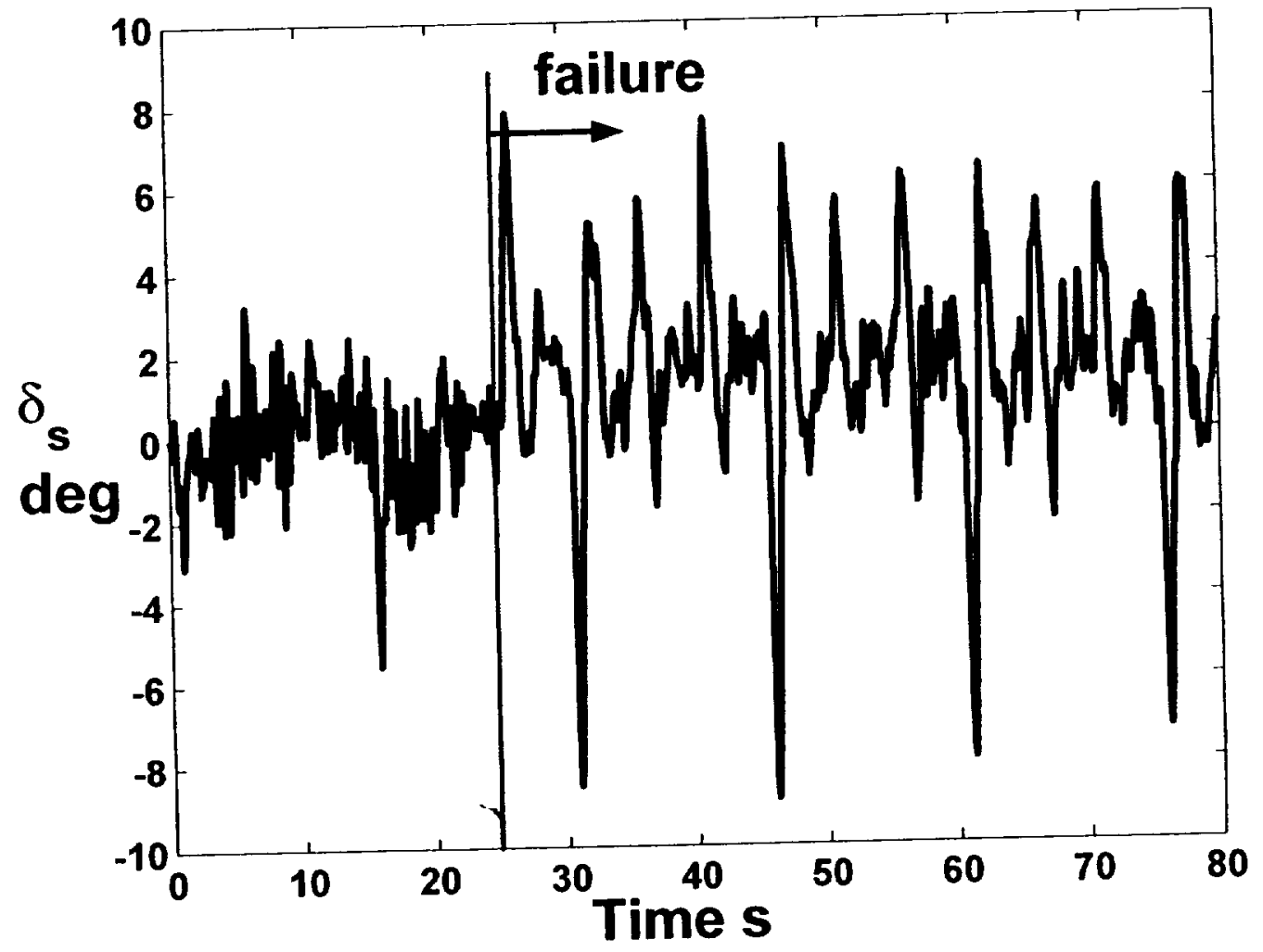

Figure 17 SMC stabilator time history before and after Failure No. 1 
$\frac{3}{\infty}$

용

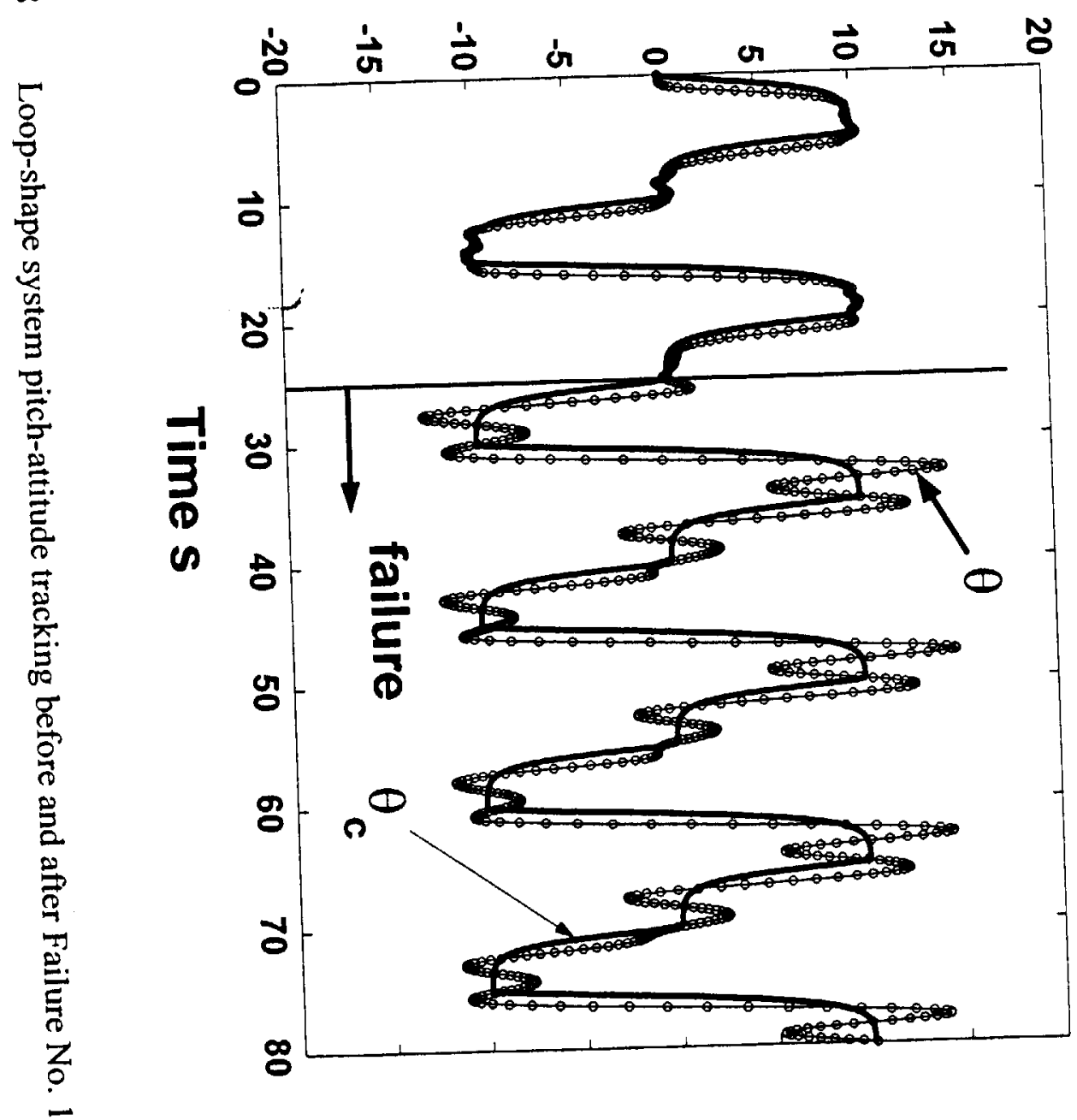




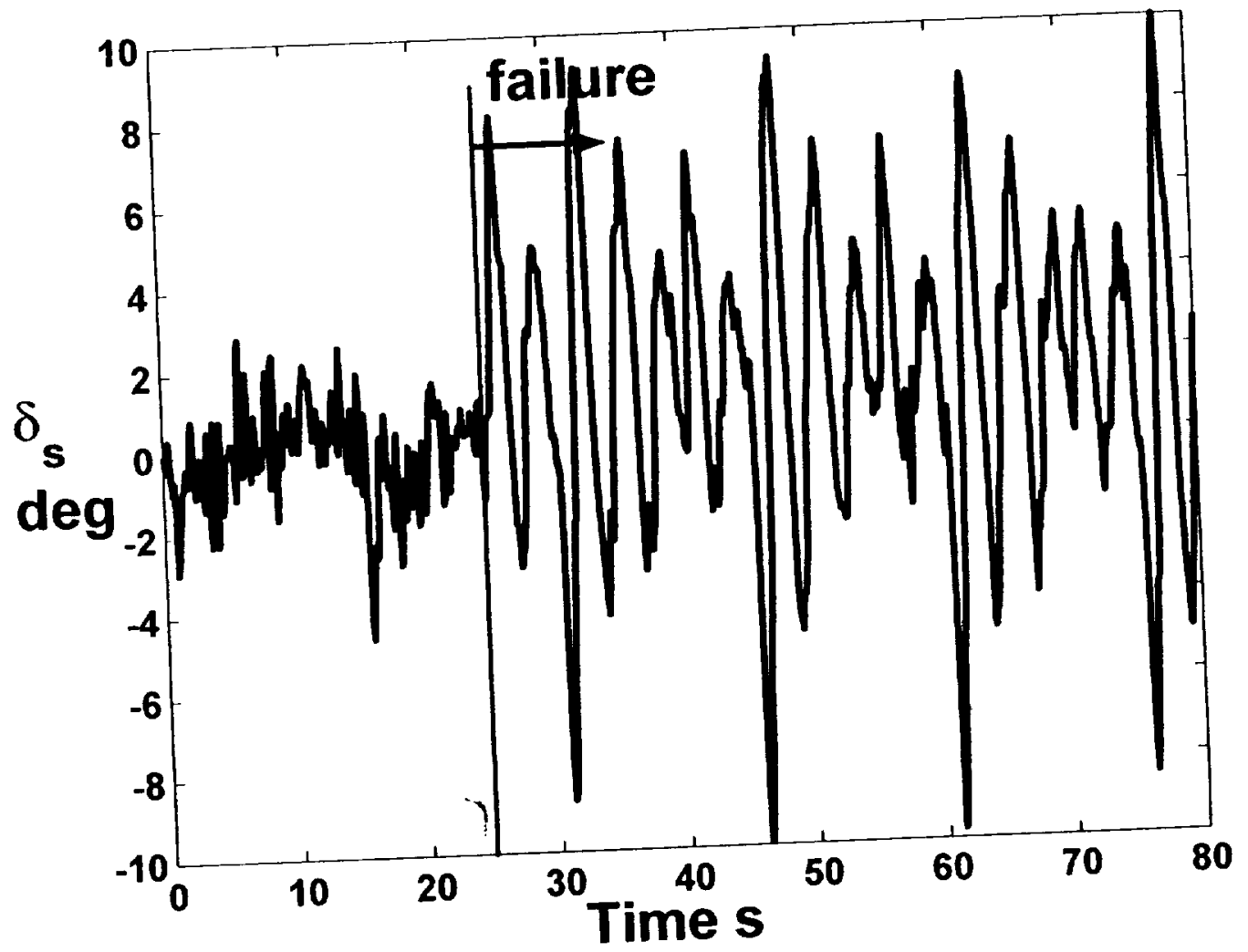

Figure 19 Loop-shape system stabilator time history before and after Failure No. 1 


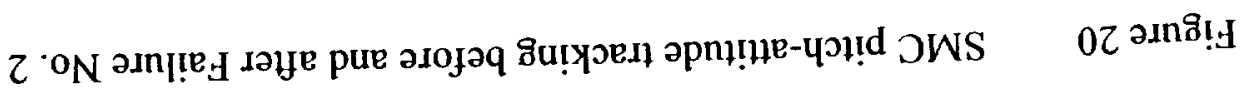

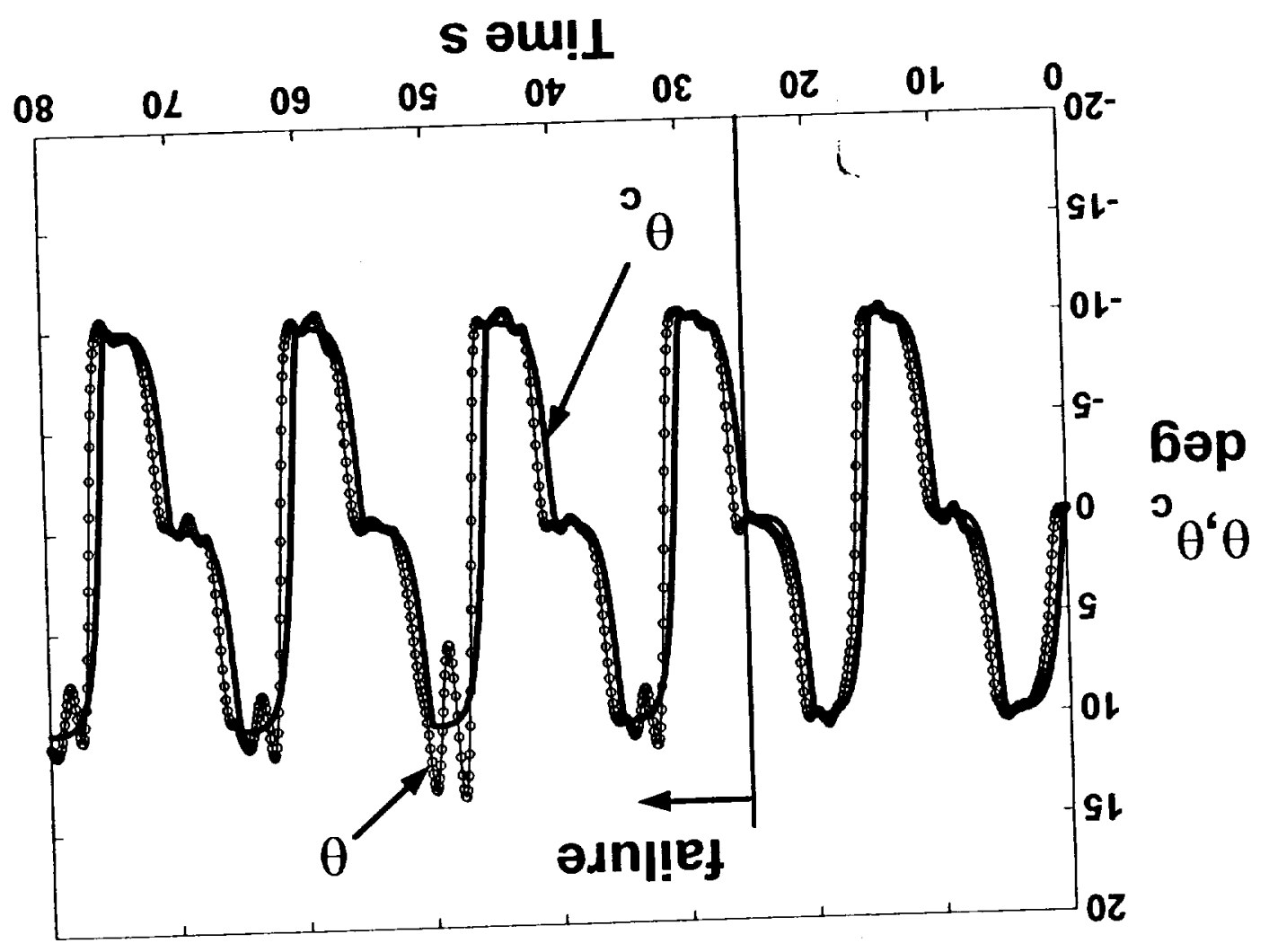




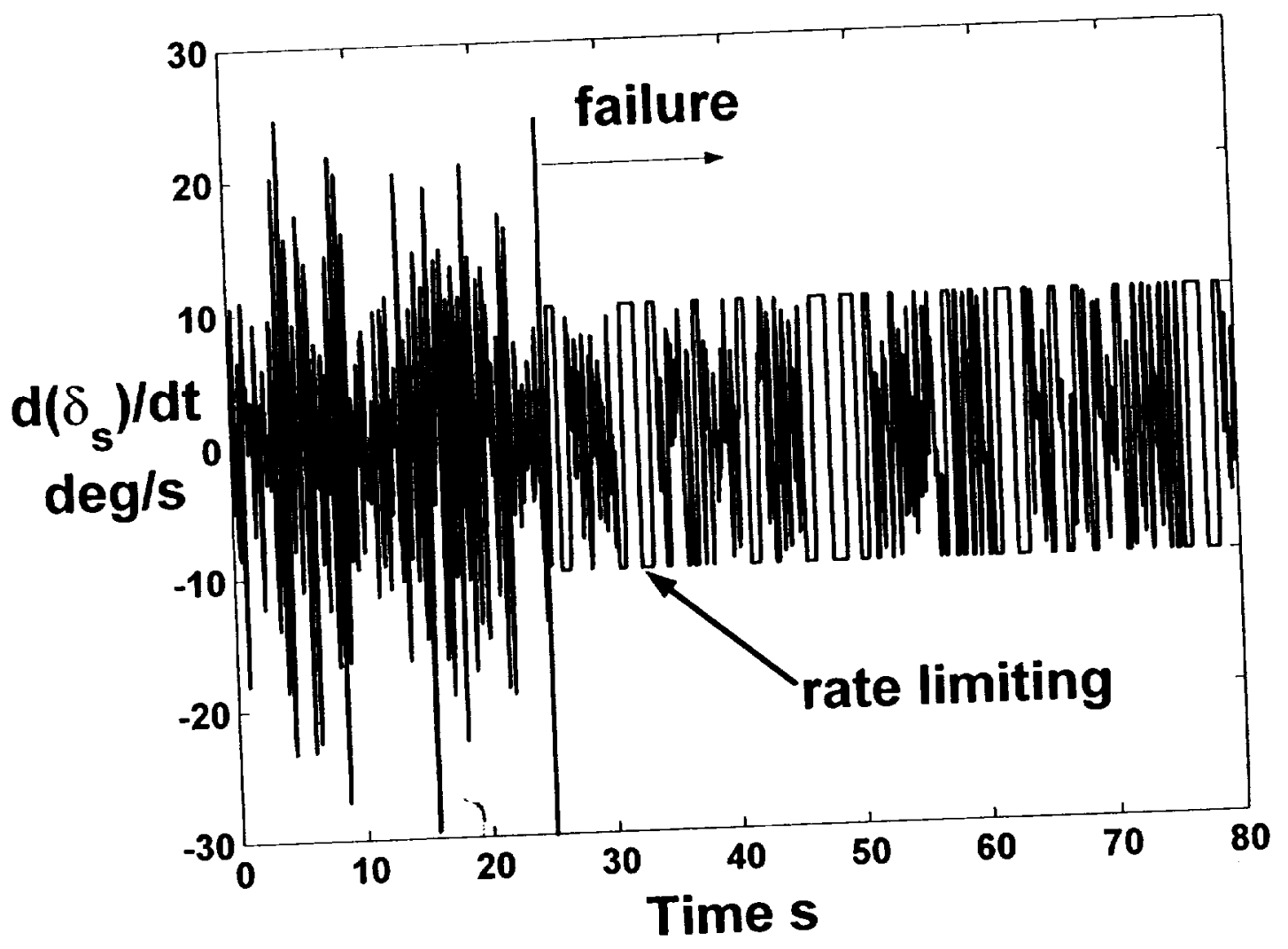

Figure 21 SMC stabilator-rate time history before and after Failure No. 2 


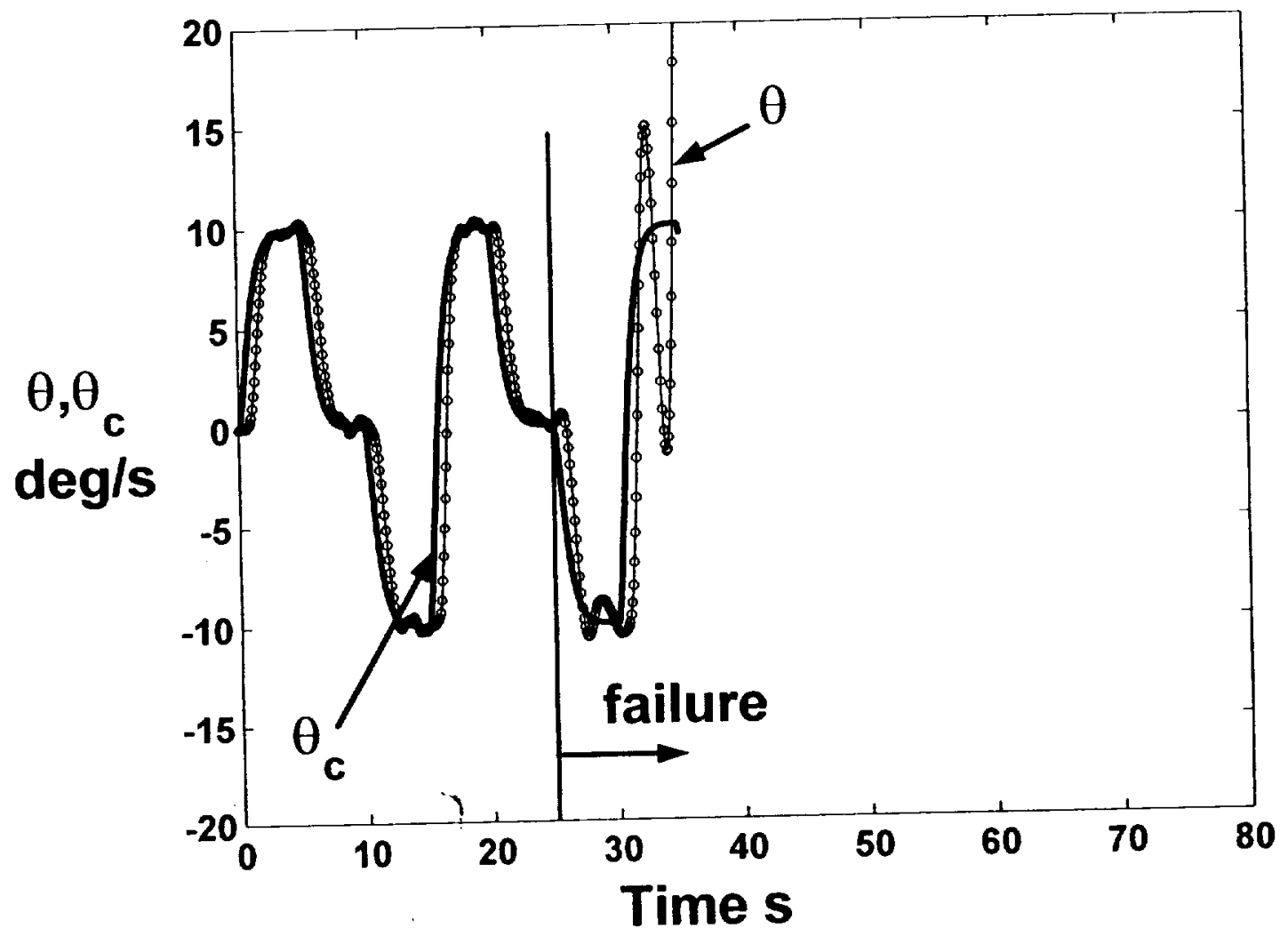

Figure 22 Loop-shape system pitch-attitude tracking before and after Failure No. 2 